\title{
Quasi-geostrophic shallow-water doubly-connected vortex equilibria and their stability
}

\author{
Hanna Plotka $†$ and David G. Dritschel \\ School of Mathematics and Statistics, University of St Andrews, St Andrews, Fife KY16 9SS, UK \\ (Received 26 November 2012; revised 29 January 2013; accepted 16 February 2013; \\ first published online 16 April 2013)
}

We examine the form, properties, stability and evolution of doubly-connected (twovortex) relative equilibria in the single-layer $f$-plane quasi-geostrophic shallow-water model of geophysical fluid dynamics. Three parameters completely describe families of equilibria in this system: the ratio $\gamma=L / L_{D}$ between the horizontal size of the vortices and the Rossby deformation length; the area ratio $\alpha$ of the smaller to the larger vortex; and the minimum distance $\delta$ between the two vortices. We vary $0<\gamma \leqslant 10$ and $0.1 \leqslant \alpha \leqslant 1.0$, determining the boundary of stability $\delta=\delta_{c}(\gamma, \alpha)$. We also examine the nonlinear development of the instabilities and the transitions to other near-equilibrium configurations. Two modes of instability occur when $\delta<\delta_{c}$ : a small- $\gamma$ asymmetric (wave 3) mode, which is absent for $\alpha \gtrsim 0.6$; and a large- $\gamma$ mode. In general, major structural changes take place during the nonlinear evolution of the vortices, which near $\delta_{c}$ may be classified as follows: (i) vacillations about equilibrium for $\gamma \gtrsim 2.5$; (ii) partial straining out, associated with the small $-\gamma$ mode, where either one or both of the vortices get smaller for $\gamma \lesssim 2.5$ and $\alpha \lesssim 0.6$; (iii) partial merger, occurring at the transition region between the two modes of instability, where one of the vortices gets bigger, and (iv) complete merger, associated with the large- $\gamma$ mode. We also find that although conservative inviscid transitions to equilibria with the same energy, angular momentum and circulation are possible, they are not the preferred evolutionary path.

Key words: contour dynamics, rotating flows, vortex dynamics

\section{Introduction}

The study of two-dimensional vortex dynamics has received considerable attention over the past few decades, largely because of its relevance to geophysical flows. It has been approached from two different perspectives, the first of which has been to directly examine the vortex-dominated processes in realistic geophysical phenomena (e.g. Hoskins, McIntyre \& Robertson 1985; McIntyre 1993; Carton 2001). The second has arisen from the study of two-dimensional turbulence which, especially at late times, is dominated by widely-separated vortex structures within a sea of filamentary debris (see e.g. Fornberg 1977; McWilliams 1984; Santangelo, Benzi \& Legras 1989; Dritschel et al. 2008, and references therein).

Understanding vortex interactions both qualitatively and quantitatively is key in both of these approaches. The majority of studies performed to date have concentrated on 
vortices of equal size and strength (Zabusky, Hughes \& Roberts 1979; Saffman \& Szeto 1980; Polvani, Zabusky \& Flierl 1989; Waugh 1992; Dritschel 1986, to name a few). However, exact symmetry is rare in realistic flows, and even slight departures from it have been shown to change the nature of the vortex interactions (see $\S 5.1$ of Dritschel 1995). A few studies have examined the asymmetry in size (Melander, McWilliams \& Zabusky 1987; Dritschel \& Waugh 1992; Dritschel 1995; Mitchell \& Driscoll 1996), strength (Yasuda \& Flierl 1995), or both (Trieling, Fuentes \& van Heijst 2005; Makarov \& Kizner 2011), and, in short, the variety of different forms of behaviour is remarkably rich.

The theory of 'adiabatic steadiness' (see Legras, Dritschel \& Caillol 2001, and references) states that widely separated vortices evolve through a series of nearequilibrium states in between strong interactions. These states are determined by the instantaneous local straining flow exerted by the surrounding vortices, and the evolution takes place until an unstable equilibrium state is reached, after which rapid, unsteady motion of the vortices ensues. Hence, according to this theory, understanding the solutions and stability of the equilibrium states, which are states that do not change in shape in an appropriately chosen frame of reference, allows predictions as to the nature of vortex interactions.

The study of relative vortex equilibria (or just 'equilibria') dates back to Deem \& Zabusky (1978a,b), who studied single (simply-connected) rotating vortex equilibria and two-vortex (doubly-connected) translating equilibria. Since then, various other studies have examined the problem, including: Saffman \& Szeto (1980), who found steady solutions for symmetric co-rotating states; Pierrehumbert (1980), who did the same for symmetric translating states; Dritschel $(1985,1995)$ who examined 2-8 like-signed vortex equilibria and asymmetric like- and opposite-signed vortices, respectively; and Makarov \& Kizner (2011) who studied two-vortex equilibria of unequal sizes and vorticities.

A limitation of the aforementioned studies is that they deal exclusively with twodimensional (or 'barotropic') systems, while in realistic geophysical flows stratification also plays an important dynamical role. As a midpoint between the two-dimensional and the more complex three-dimensional systems, the quasi-geostrophic shallow-water (QGSW) approximation may be used. Through the introduction of a new length scale, the Rossby deformation length $L_{D}=c / f$, a link can be made between the effects of stratification (represented by the short-scale gravity wave speed $c$ ) and planetary rotation (represented by the Coriolis frequency $f$ ) in a succinct way. Fluid motions having scales much smaller than $L_{D}$ behave in the classical two-dimensional manner, whereas motions at scales larger than $L_{D}$ become confined to fronts or jets of width $O\left(L_{D}\right)$ and are strongly affected by free-surface or layer-thickness deformations.

Only a few studies have examined the effects of a finite Rossby deformation length on vortex equilibria. Yet both experimental (Griffiths \& Hopfinger 1986, 1987) and numerical studies have found qualitative changes in the behaviour of the system. Polvani (1988) and Polvani et al. (1989) have studied equilibrium forms of simply-connected and doubly-connected states, and Płotka \& Dritschel (2012) have additionally examined the linear and nonlinear stability of simply-connected states. Waugh (1992) examined the effects of $L_{D}$ on symmetric vortex merger, and Yasuda (1995) showed that the QGSW model better represents realistic oceanic vortices. These studies have found that larger vortices are able to sustain greater deformations and get closer together before the onset of instability than smaller ones, and that at large scales (larger than $L_{D}$ ) there is a roll-up of filaments of vorticity and a suppression of filamentation. Makarov, Sokolovskiy \& Kizner (2012) examined the effects of $L_{D}$ 
on the form and stability of a pair of steadily translating, doubly-symmetric vortex patches, where each patch was located in a different layer of a two-layer rotating fluid. They found that increasing the size of this two-vortex structure relative to $L_{D}$ destabilizes it, although for large enough vortices the conditions necessary for stability become $L_{D}$-independent. Other studies, including Waugh \& Dritschel (1991) who studied generalized geophysical models of vortex dynamics, further elaborate the differences between the large- and small- $L_{D}$ regimes.

The goal of the present study is to extend the work of Dritschel (1995) and Polvani et al. (1989). The former studied two-dimensional asymmetric doubly-connected equilibria, and examined their linear and nonlinear stability. The latter examined the form of symmetric doubly-connected states at finite $L_{D}$ and found the critical distance between the two vortices for merger to occur. We go further and examine the effects of finite $L_{D}$ on the form, properties, linear and nonlinear stability of doubly-connected co-rotating vortex equilibria, aiming also to quantify and describe their nonlinear interactions.

The paper is organised as follows. In $\S 2$ we present the theoretical and numerical framework for generating the equilibria, and discuss their form and properties; in $\S 3$ we perform a full linear stability analysis of the equilibria, and in $\S 4$ we examine their nonlinear stability and evolution. In $\S 5$ we examine the transitions between simplyand doubly-connected equilibria, and finally we draw some conclusions in $\S 6$.

\section{Flow model and properties of the equilibria}

\subsection{Quasi-geostrophic shallow-water flow and the vortex-patch model}

The quasi-geostrophic shallow-water model (cf. Pedlosky 1979) has been widely applied to study fundamental features of atmospheric and oceanic dynamics. It describes the effects of both rotation and stratification, which are central to geophysical flows. Its simplifying feature is that in addition to the non-divergent flow field $\boldsymbol{u}=(u, v)$ being two-dimensional, the evolution of the system is governed by the material advection of a single scalar, namely the quasi-geostrophic potential vorticity (PV) $q$,

$$
\left(\frac{\partial}{\partial t}+\boldsymbol{u} \cdot \nabla\right) q=0,
$$

where the PV is related to the streamfunction $\psi$ and $\boldsymbol{u}=(-\partial \psi / \partial y, \partial \psi / \partial x)$ by

$$
q=\left(\nabla^{2}-L_{D}^{-2}\right) \psi .
$$

By considering the single-layer form of the model on the $f$-plane, we look for equilibrium solutions of two vortex patches of uniform and equal PV $q_{1}=q_{2}=q_{0}$ in an unbounded domain having $q=0$, as shown in figure 1. Although very simple in form, vortex patches are a good approximation of realistic high-Reynolds-number flows, as these commonly exhibit vortices with steep PV gradients on their boundaries, having near-uniform values of PV within their core (see e.g. Melander et al. 1987; Dritschel 1993; Legras \& Dritschel 1993). By employing a formulation now termed 'contour dynamics', Deem \& Zabusky $(1978 a, b)$ showed that the dynamics of vortex patches, which can be determined from the superposition of their induced velocities, is determined wholly by the shape of their bounding contours. For $k$ regions of constant potential vorticity in irrotational flow, the velocity field is determined from the contour 


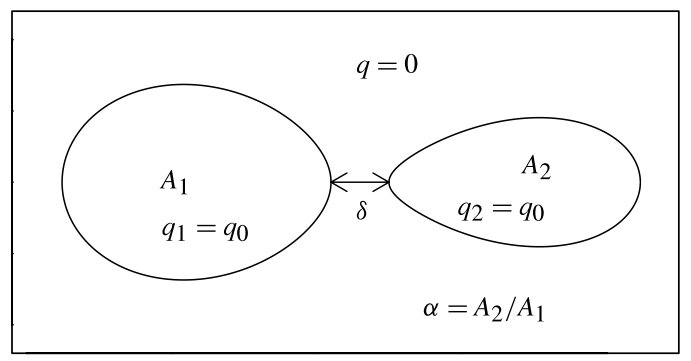

FIGURE 1. Schematic diagram of the doubly-connected equilibria.

integral

$$
\frac{\mathrm{d} \boldsymbol{x}}{\mathrm{d} t}=\boldsymbol{u}(\boldsymbol{x})=\sum_{k} \frac{q_{k}}{2 \pi} \oint_{\mathscr{C}_{k}} \mathrm{~K}_{0}\left(\left|\boldsymbol{x}-\boldsymbol{x}_{k}\right| / L_{D}\right) \mathrm{d} \boldsymbol{x}_{k},
$$

where $\mathrm{K}_{0}$ is the modified Bessel function of zeroth-order, $\mathscr{C}_{k}$ is the bounding contour of each patch $k$, drawn in a right-handed sense, $q_{k}$ is the inward jump of PV across $\mathscr{C}_{k}$, and $\boldsymbol{x}_{k} \in \mathscr{C}_{k}$, as generalized to multiple contours by Dritschel (1989). This is obtained by using Stokes' theorem on the double integral involved in inverting $\nabla^{2}-L_{D}^{-2}$ in (2.2). When $\boldsymbol{x} \in \mathscr{C}_{j}$ also, this equation fully describes the motion of each contour $\mathscr{C}_{j}$.

We set $k=2$ in (2.3) and seek families of asymmetric doubly-connected equilibria. In this configuration, the families are completely spanned by three dimensionless parameters: (i) their size relative to the Rossby deformation length $\gamma=L / L_{D}$; (ii) the ratio between the areas of the smaller and the larger vortex $0<\alpha=A_{2} / A_{1} \leqslant 1$; and (iii) the distance $\delta$ between the innermost edges of the vortices.

Using a modification (Dritschel 1985, 1995) of the iterative scheme first used by Pierrehumbert (1980), we generate asymmetric doubly-connected co-rotating vortex equilibria. Without loss of generality, we set $q_{0}=1$ (giving $q_{1}=q_{2}=1$ ) and $A=A_{1}+A_{2}=\pi$, where $A_{1} \geqslant A_{2}$. This prescribes the areas $A_{1}=\pi /(1+\alpha)$ and $A_{2}=\alpha \pi /(1+\alpha)$ of each of the vortices in terms of the area ratio between them. Throughout this paper the sub- and superscripts 1 and 2 will be used to distinguish between the larger and smaller vortices, which, without loss of generality, in equilibrium we set to be on the left-hand and right-hand side of the state, respectively (cf. figure 1).

For each $\alpha$, we begin the iterative procedure with a first guess for the equilibrium shapes, namely two circular vortices at a sufficiently large distance $\delta$ apart, proportional to $2 / \gamma$. For this guess we approximate the rotation rate of the equilibrium to be that of two point vortices located at the centre of each patch, namely $\Omega=\gamma \mathrm{K}_{1}(\gamma d) / 2 d$, where $\mathrm{K}_{1}$ is the modified Bessel function of the first-order and $d=\delta+r_{1}+r_{2}$ is the distance between the centroids of the vortices. We then progressively decrease the distance $\delta$ by $\Delta \delta=0.001$, at each step finding a solution for the boundaries of the vortices and the rotation rate $\Omega$ until convergence, here when the maximum normal variation of the contour shapes is less than $10^{-7}$. We continue this procedure until we reach a final distance $\delta_{f}$ after which we either fail to obtain convergence, or a numerical artefact in the form of a 'spike' appears (see Wu, Overman \& Zabusky 1985 for more details on this). The value of $\delta_{f}$ is dependent on both $\gamma$ and $\alpha$ : as an example, for $\alpha=0.2$ for various $\gamma$, we find $0.013 \leqslant \delta_{f} \leqslant 0.647$ (with $\delta_{f}$ decreasing with increasing $\gamma$ ), while for $\alpha=1.0$, we find $\delta_{f}=0.001$ for all $\gamma$. 
(a)

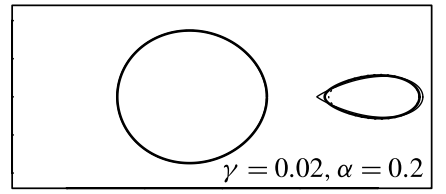

(d)

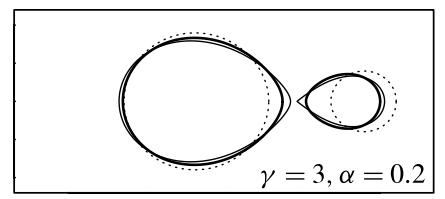

$(g)$

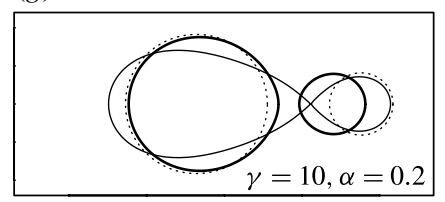

(b)

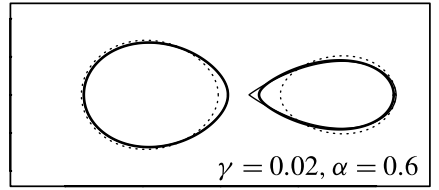

(e)

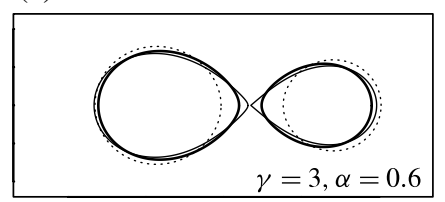

(h)

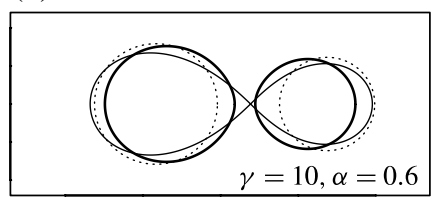

(c)

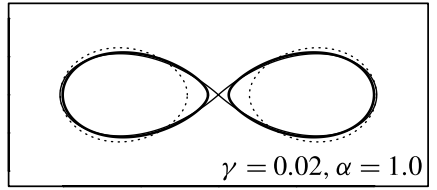

$(f)$

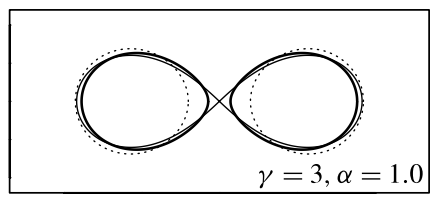

(i)

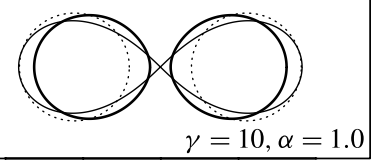

FIGURE 2. Examples of doubly-connected vortex equilibria. For each case we show the distances $\delta=0.8$ (dashed line), the critical distance $\delta=\delta_{c}$ (bold line), and the smallest distance attained $\delta=\delta_{f}$ (thin line). Note, $|x| \leqslant 2.7,|y| \leqslant 1.2$. In these and subsequent figures we are in a frame of reference rotating with the equilibria. See also supplementary movie 1 available at http://dx.doi.org/10.1017/jfm.2013.104 for the case $\alpha=0.2$.

To obtain the high levels of accuracy in the equilibrium shapes necessary for solving the linear stability problem (see §3), we use 200 nodes to represent half of the boundary of each of the vortices. Note that the iterative scheme fixes the global centroid of the equilibria at $(x, y)=(0,0)$, and enforces symmetry about the $y$-axis, so that we have to compute only half of the boundary of each vortex.

We seek families of doubly-connected equilibria for a range of $\alpha$ and $\gamma$. We generate barotropic-like families with small $\gamma=0.02,0.25$ and 0.5 , and families which increasingly depart from barotropy $(\gamma=0)$ with $\gamma=1-3.5$ at $\Delta \gamma=0.5$ and $\gamma=4-10$ at $\Delta \gamma=1$. A denser range of families is necessary at smaller $\gamma$, as the equilibria depart from barotropy quickly, but once a critical value of $\gamma \approx 3$ is reached, differences between them are small. The range of $\gamma$ examined provides a good description of the variety of motions found in geophysical contexts (Charney \& Flierl 1981; Carton 2001; Scott \& Polvani 2008). For each $\gamma$, we examine $\alpha=0.1-1.0$ at $\Delta \alpha=0.1$. Note that Dritschel (1995) has studied in detail the case $\gamma=0$, which is omitted in this study (we instead examine $\gamma=0.02$ for comparison), for a range of $\alpha$, while Polvani et al. (1989) examined families of equilibria for a range of $\gamma$ at $\alpha=1.0$, but did not address the problem of their stability.

\subsection{Properties of the equilibria}

Several examples of how equilibrium contour shapes change with $\gamma$ and $\alpha$ are shown in figure 2 (and in the supplementary movie 1). For each value of $\gamma(0.02,3$ and 10 ), we show three different $\alpha$ : a highly-asymmetric state with $\alpha=0.2$, a moderatelyasymmetric state with $\alpha=0.6$, and a symmetric state with $\alpha=1.0$. For each $(\gamma, \alpha)$, we show three distances: a 'large' distance $\delta=0.8$, the critical distance $\delta=\delta_{c}$ at the boundary of stability (see $\S 3$ ), and the smallest distance $\delta=\delta_{f}$ for which we achieve 
convergence. We see that as the distance decreases, the vortices become increasingly deformed, until either one or both of them develop a sharp corner. For fixed $\gamma$, as $\alpha$ increases, the vortices are able to move closer and closer together before either $\delta_{c}$ or $\delta_{f}$ is reached. Also, the smaller vortex tends to be more deformed than the larger one, with this most pronounced for small $\alpha$. Deformations of equal-sized vortices are symmetric. As $\gamma$ increases both $\delta_{c}$ and $\delta_{f}$ decrease, and so despite the weakening of long-range interactions, even for small $\alpha$ the shapes of both of the vortices are affected by each others' presence. Across all $\alpha$, we see that when $\delta=0.8$, the vortices are nearly unaffected by each other for $\gamma=3$ and 10, in contrast to what is seen from the elongated form of the vortex at $\gamma=0.02$. For the $\gamma=10$ equilibria, even at $\delta=\delta_{c}$ the shape of each vortex is hardly changed from a circular form. At $\delta=\delta_{f}$, despite having moved closer together at a single point, the larger vortex appears to push the smaller one away, while at the same time deforming considerably.

Further properties of the equilibria are exhibited in figure 3 for each of the five area ratios, $\alpha=0.2,0.4,0.6,0.8$ and 1.0 (rows $a-e$ of the figure, respectively) in the $\gamma-\delta$ parameter plane. In the first column we show the angular impulse $J=q_{0} \iint_{\mathscr{D}}\left(x^{2}+y^{2}\right) \mathrm{d} x \mathrm{~d} y$, where $\mathscr{D}$ is the region inside the vortex patches, in the second column the total energy $E=-\left(q_{0} / 2\right) \iint_{\mathscr{D}} \psi \mathrm{d} x \mathrm{~d} y$, and in the third column the rotation rate $\Omega$. Note that the barotropic families of equilibria are not included, and instead the nearly barotropic case $\gamma=0.02$ is used.

We first examine $J$ and $E$. For each $\gamma$ considered we find that there is a distance for which $J$ exhibits a minimum, and an almost identical distance at which $E$ exhibits a maximum, marked by the bold dashed lines in the figure. The biggest difference between the two extrema ranges between 0.009 and 0.012 , depending on $\alpha$. As shown by Luzzatto-Fegiz \& Williamson (2012), the location of the extrema of $J$ and $E$ must be the same, and we expect it to coincide with the location of the boundary of stability (see discussion below). In this study, the difference in $\delta$ at which the extrema occur is due to the fact that the function of $E(\delta)$ is very flat (cf. column 2 of figure 3 ), implying that it is very sensitive to finding the exact location of the maximum. On the other hand, the location of the minimum of $J$ is better defined, and thus provides a more trustworthy value for the boundary of stability (see figure 4). Figure 4 shows how $J$ changes with $\alpha$ for $\gamma=0.02,0.5,1,2,4$ and 10 . It can be seen that as $\gamma$ increases, the location of the minimum of $J$ (indicated by a dashed line in the figure) becomes independent of $\alpha$, and the minimum itself becomes more obvious. It appears that the numerical method fails to converge at the point when $J$ starts levelling off after its minimum for small $\delta$. This is not only more pronounced for larger $\gamma$, but also occurs at smaller distances for small $\alpha$. The angular impulse increases with $\alpha$, most strongly for small $\gamma$. However, the degree of asymmetry (given by $\alpha$ ) does not affect $J$ linearly; in fact, regardless of $\gamma$, states with $\alpha=0.8$ and 1.0 have very similar values of $J$, while there is a considerable discrepancy between the values of $J$ for $\alpha=0.2-0.6$.

Saffman \& Szeto (1980) and Saffman (1992) argued that when the plot of $E(J)$ has the form of two branches of solutions joined at a cusp (i.e. when there exist simultaneous extrema of $J$ and $E$ ), the lower branch is unstable whereas the upper one is linearly stable. Dritschel (1985) showed that this argument is not complete, and Dritschel (1995) demonstrated that the joint extremum of $J$ and $E$ coincides with the boundary of stability only for like-signed pairs of barotropic $(\gamma=0)$ vortices of equal or unequal size. Figure 5 shows a plot of $E$ as a function of $J$ for the $\gamma=2$, $\alpha=0.2,0.6,1.0$ families of equilibria (all other families are qualitatively similar, with the length of the lower branch increasing with $\gamma$ ). We see that the plots have the form 
(a)

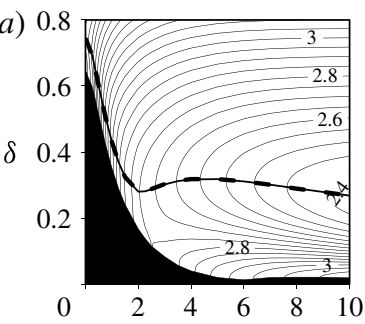

(b) 0.8

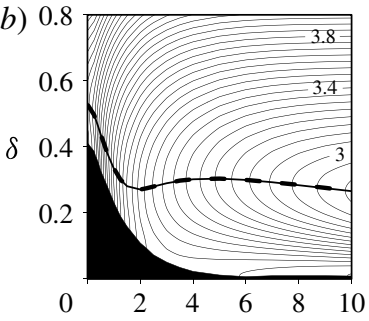

(c)

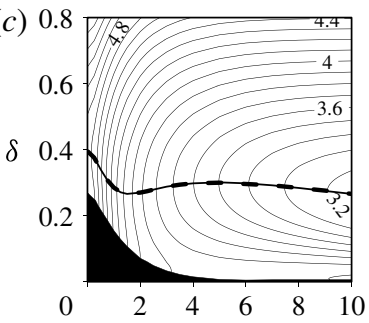

(d) 0.8

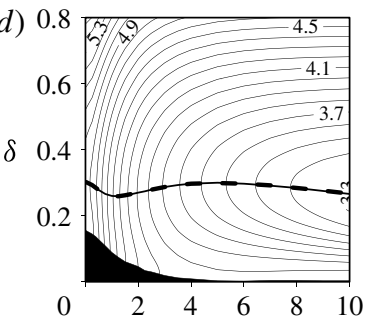

(e)

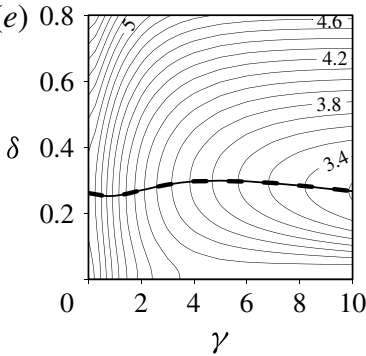

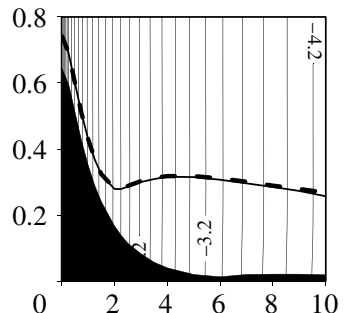
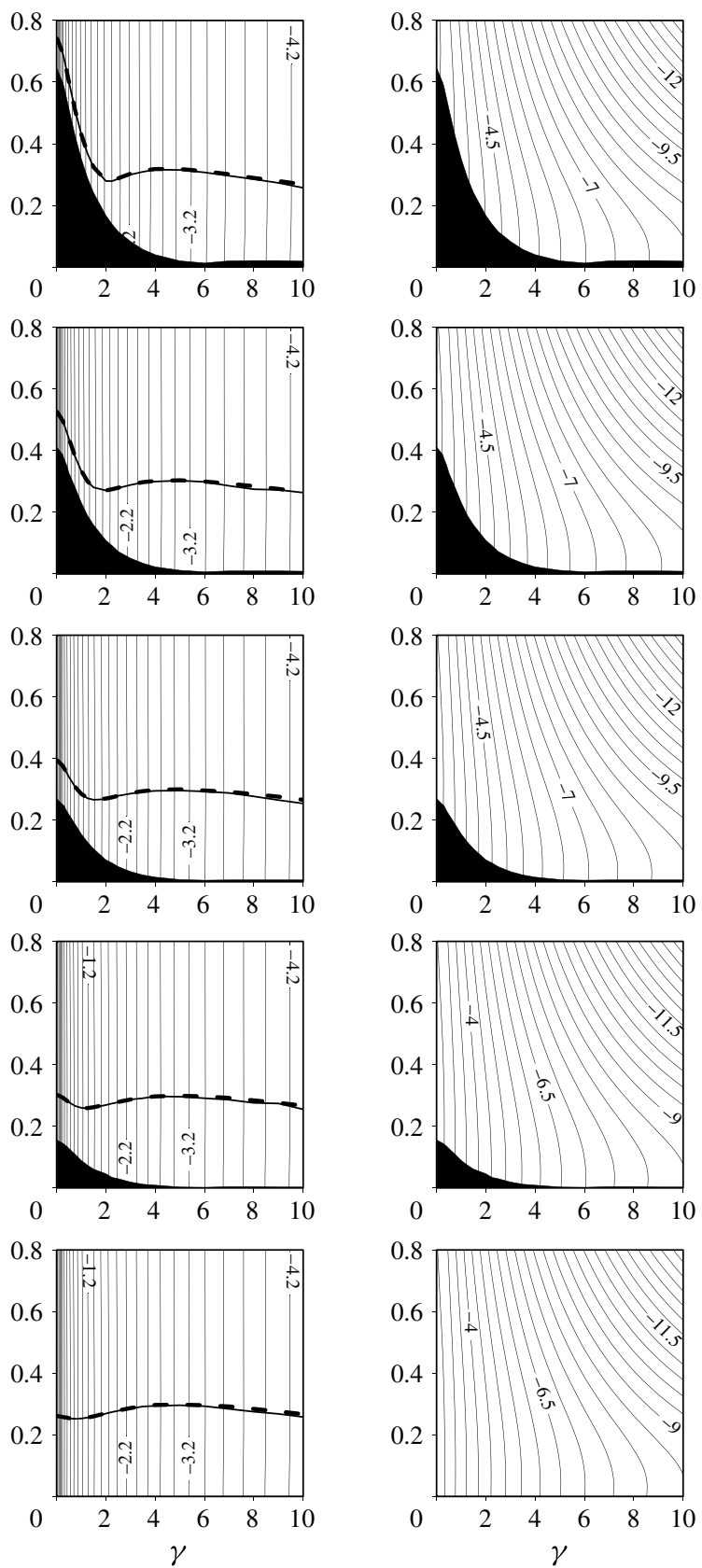

FIGURE 3. Various properties of the equilibria in the $\gamma-\delta$ parameter plane for five different area ratios $\alpha=0.2,0.4,0.6,0.8$ and 1.0 (rows $a-e$, respectively): angular impulse $J$ (first column, contour interval 0.05 for rows $a, b$ and 0.1 for rows $c-e$ ), energy $E$ (second column, $\log _{10}$ scaling, contour interval 0.2), and rotation rate $\Omega$ (third column, $\log _{10}$ scaling, contour interval 0.5 ). The bold dashed line corresponds to the boundary of linear stability (with instability below, see $\S 3$; note that this very closely coincides with the boundary of nonlinear stability, see § 4.2) and the bold line marks the extrema of $J$ or $E$ with respect to $\delta$ for each $\gamma$. In this and in figures 8,17 and 19 , the black area is the region for which no equilibria have been generated. 

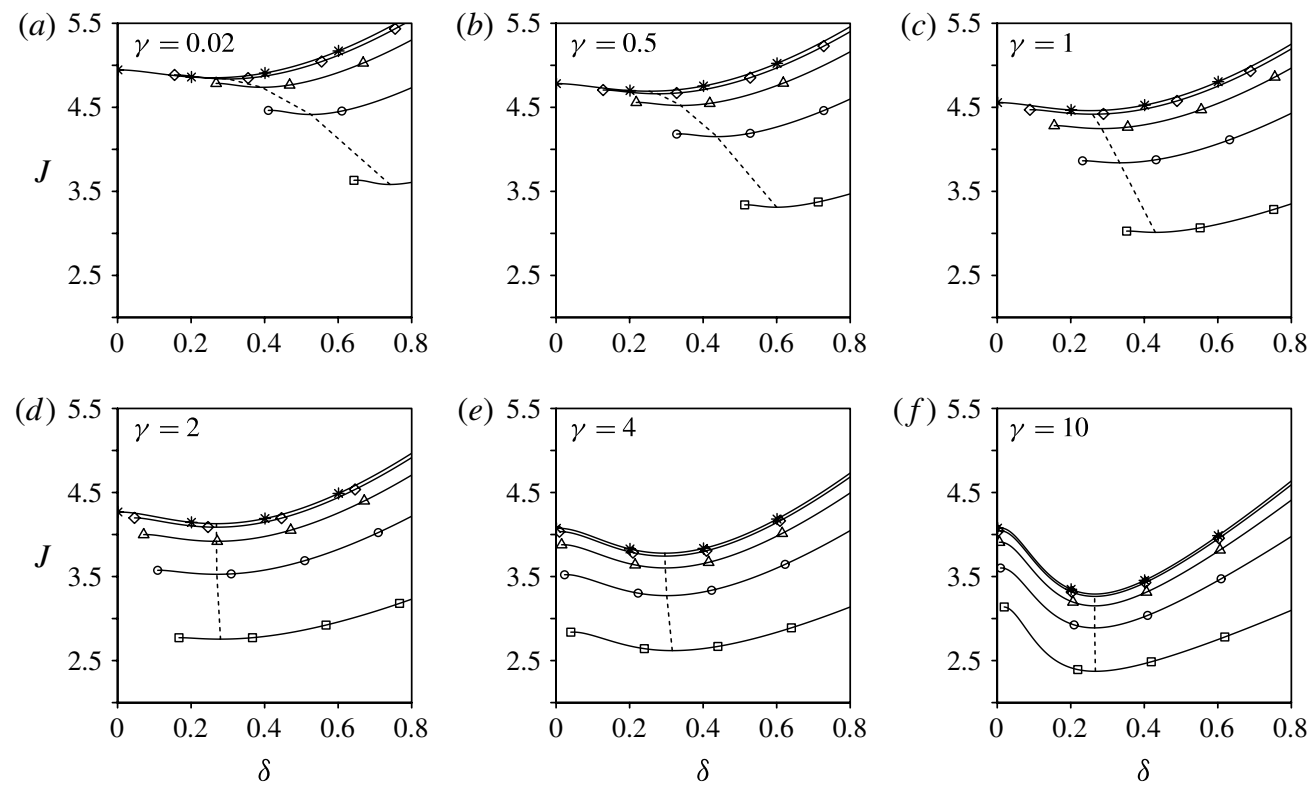

FIGURE 4. The angular impulse $J$ against distance for various $\gamma$. The dashed line connects the minima of $J$, and the symbols on each line represent different area ratios: $\square, \alpha=0.2$; $\bigcirc, \alpha=0.4 ; \triangle, \alpha=0.6 ; \diamond, \alpha=0.8 ; *, \alpha=1.0$.
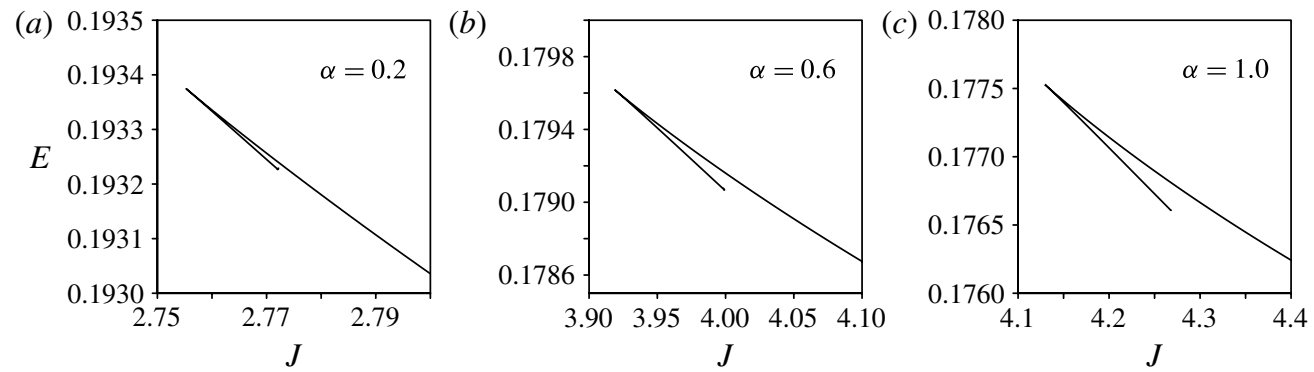

FIGURE 5. The energy $E$ as a function of angular impulse $J$ at $\gamma=2$ for three different $\alpha$.

described above. The linear stability analysis below confirms the coincidence of the boundary of linear stability with extrema of $J$ and $E$ also when $\gamma \neq 0$ (see $\S 3$, and the bold dashed line in figure 3). The nonlinear stability analysis (see $\S 4$ ) also finds the boundary of nonlinear stability to fall at this location.

Figure 6 shows how the particle rotation rates $\Omega_{p, i}=2 \pi / T_{i}$, where $T_{i}=\oint_{\mathscr{C}_{i}} \mathrm{~d} s /|\boldsymbol{u}|$ and $s$ is arclength, change with $\gamma$ and $\alpha$ for each of the vortices. We examine the same families of equilibria as those shown in figure 4, namely the cases with $\gamma=0.02,0.5$, 1, 2, 4 and 10. We see that both $\Omega_{p, 1}$ and $\Omega_{p, 2}$ decrease strongly with $\gamma$, and to a lesser extent with $\delta$. At smaller $\gamma, \Omega_{p, 1}$ of the larger vortex (solid line in figure 6) is bigger than $\Omega_{p, 2}$ of the smaller vortex (dashed line in the figure), with this being most pronounced at small distances. Note that in this regime there is a steep rise in $\Omega_{p, 2}$ for distances near the smallest distance attainable. As $\gamma$ increases, so does $\Omega_{p, 2}$ and it goes through a transition where $\Omega_{p, 2}>\Omega_{p, 1}$ at large distances and $\Omega_{p, 2}<\Omega_{p, 1}$ at small 

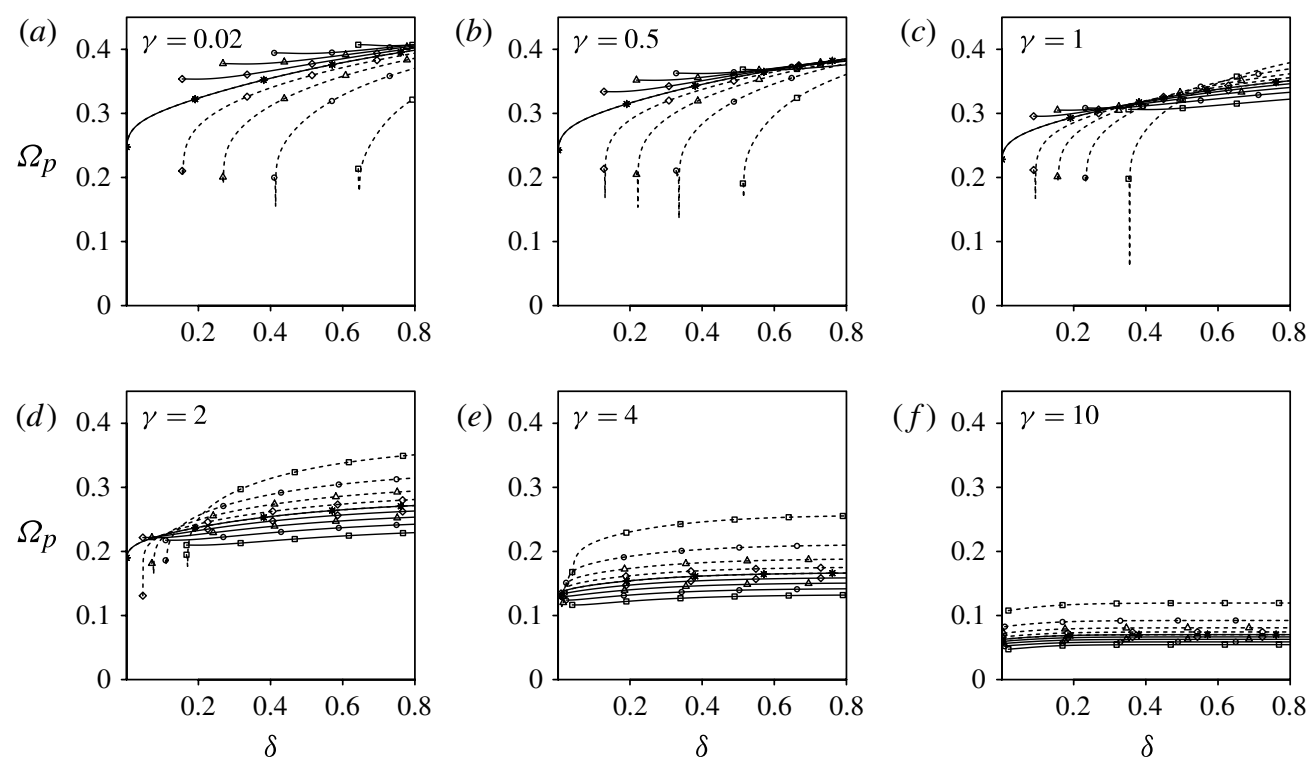

FIGURE 6. The particle frequency $\Omega_{p}$ against distance for various $\gamma$. The solid and dashed lines are $\Omega_{p}$ for the larger and smaller vortices, respectively. The symbols on each line represent different area ratios: $\square, \alpha=0.2 ; \bigcirc, \alpha=0.4 ; \triangle, \alpha=0.6 ; \diamond, \alpha=0.8 ; *, \alpha=1.0$.

ones, until at large $\gamma$, we find $\Omega_{p, 2}>\Omega_{p, 1}$ for all distances. For large $\gamma, \Omega_{p, 1}$ and $\Omega_{p, 2}$ become distance-independent, as discussed below.

By comparing column 3 of figure 3 with figure 6, we see that like the particle rotation rates, the rotation rate $\Omega$ also decreases strongly with $\gamma$, and to a lesser extent with $\delta$. We further note that for large $\gamma$, we have $\Omega_{p} \gg \Omega$. Here, the fluid velocity induced by each of the vortices is confined to a belt of $O\left(L_{D}\right)$ width, where the leading-order velocity on each contour is $\sim q_{0} L_{D} / 2$ (Nycander, Dritschel \& Sutyrin 1993). This in turn implies that $\Omega_{p, i} \sim q_{0} L_{D} / 2 P_{i}$, where $P_{i}$ is the perimeter of vortex $i$. This relationship holds within $10 \%$ for both vortices across all $\alpha$ at $\gamma=10$. In general, the relationship is more accurate for the larger vortex at small $\alpha$, and for the smaller vortex at large $\alpha$. The equilibrium rotation rate $\Omega$, on the other hand, is much smaller in magnitude because it arises from weak long-range interactions associated with the Green function. If following Nycander et al. (1993) we view the deformations of each contour as long waves, then it follows that they must rotate at a frequency proportional to $\gamma^{3}$. For the equilibrium to be steady, therefore, the frame of reference must also rotate at this rate: $\Omega \approx a \gamma^{3}$. Figure 7 verifies that this scaling holds closely for $\delta=0.700$, with $a$ varying only slightly from 0.0757 (at large $\alpha$ ) to 0.0774 (at small $\alpha)$.

Note that our results for the symmetric case $\alpha=1.0$ agree with those found by Polvani et al. (1989).

\section{Linear stability analysis}

We next perform a linear stability analysis of the families of equilibria described above using a method first used by Dritschel \& Legras (1991) and fully described in Dritschel (1995). In this method, we displace the equilibrium PV contours by arbitrarily infinitesimal amounts which are normal to the contour. Such disturbances 


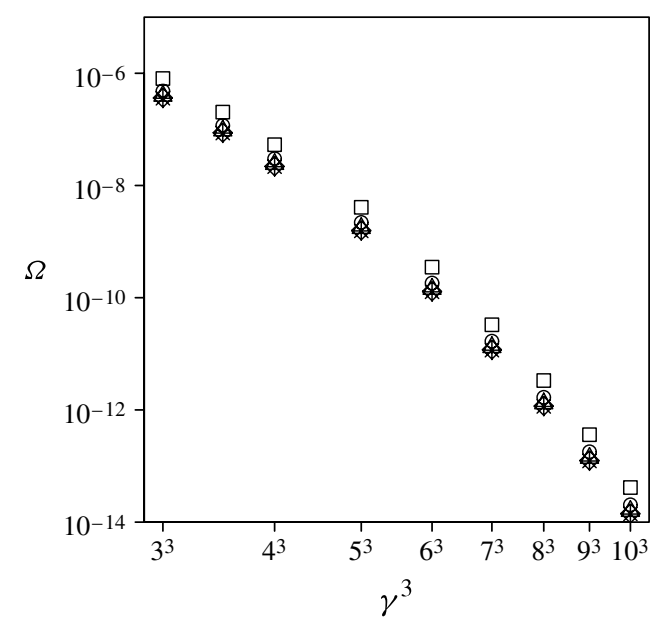

FIGURE 7. The relationship between $\Omega$ and $\gamma^{3}$ at $\delta=0.700$. The different symbols correspond to: $\square, \alpha=0.2 ; \bigcirc, \alpha=0.4 ; \triangle, \alpha=0.6 ; \diamond, \alpha=0.8 ; *, \alpha=1.0$.

can only arise under the external action of a weak irrotational field, which may for example result from some distant vortices. The disturbances are expanded in a Fourier series involving $\cos m \theta$ and $\sin m \theta$, where we retain only wavenumbers $m \leqslant M=50$, and where $\theta=\Omega_{p} t$ is an angle (measured from a point on the $x$-axis on the rightmost edge of the equilibrium contour) proportional to the travel time $t$ of a particle moving around this contour. We thus solve a $2 n M \times 2 n M$ matrix eigenvalue problem at each $\delta$ for fixed $\gamma$ and $\alpha$, where $n=N=2$ is the number of contours. We have verified that doubling the number of retained wavenumbers to $M=100$ insignificantly affects our results.

The growth rates $\sigma_{r}$ of the most unstable mode, being the real part of the solution $\sigma$ of the eigenvalue problem, are presented in figure 8 in the $\gamma-\delta$ parameter plane for each $\alpha$. The 'waviness' of the contours arises from a competition between a variety of different modes of instability; however, we are still able to distinguish a small- $\gamma$ and a large- $\gamma$ regime. At small $\gamma$, the contours of $\sigma_{r}$ are steep and growth rates rapidly decrease with $\gamma$. This is most apparent in the smaller $\alpha$ cases, with the boundary between the steep and flat contours being at $\gamma \approx 2$. As $\alpha$ increases, not only does this boundary occur at smaller $\gamma$, but also states with progressively smaller $\delta$ become stable across all $\gamma$. Note that as in $\S 2.2$, we do not show the $\gamma=0$ case, and instead use equilibria having $\gamma=0.02$; nevertheless our results converge to the barotropic results (Dritschel 1995).

Near the margin of stability, the growth rates turn out to be sensitive to the number of points $n_{p}$ chosen to discretize half of the boundary of each vortex. Figure 9 shows $\sigma_{r}$ for $n_{p}=200$ (the value used in this paper), 400 and 800. We see that as $\gamma$ increases, the results become less sensitive to the number of points. The fact that the extrema of energy $E$ and angular impulse $J$, marked by the vertical lines in the figure, do not converge may be attributed to how 'flat' the function of $E(\delta)$ is (see discussion in the previous section).

Dritschel (1995) found that for the $\gamma=0$ case, instability erupts via an 'exchange of stability', where the real and imaginary parts of $\sigma$ are both identically zero at the margin of stability $\delta=\delta_{c}$. Moreover this corresponds to simultaneous extrema 

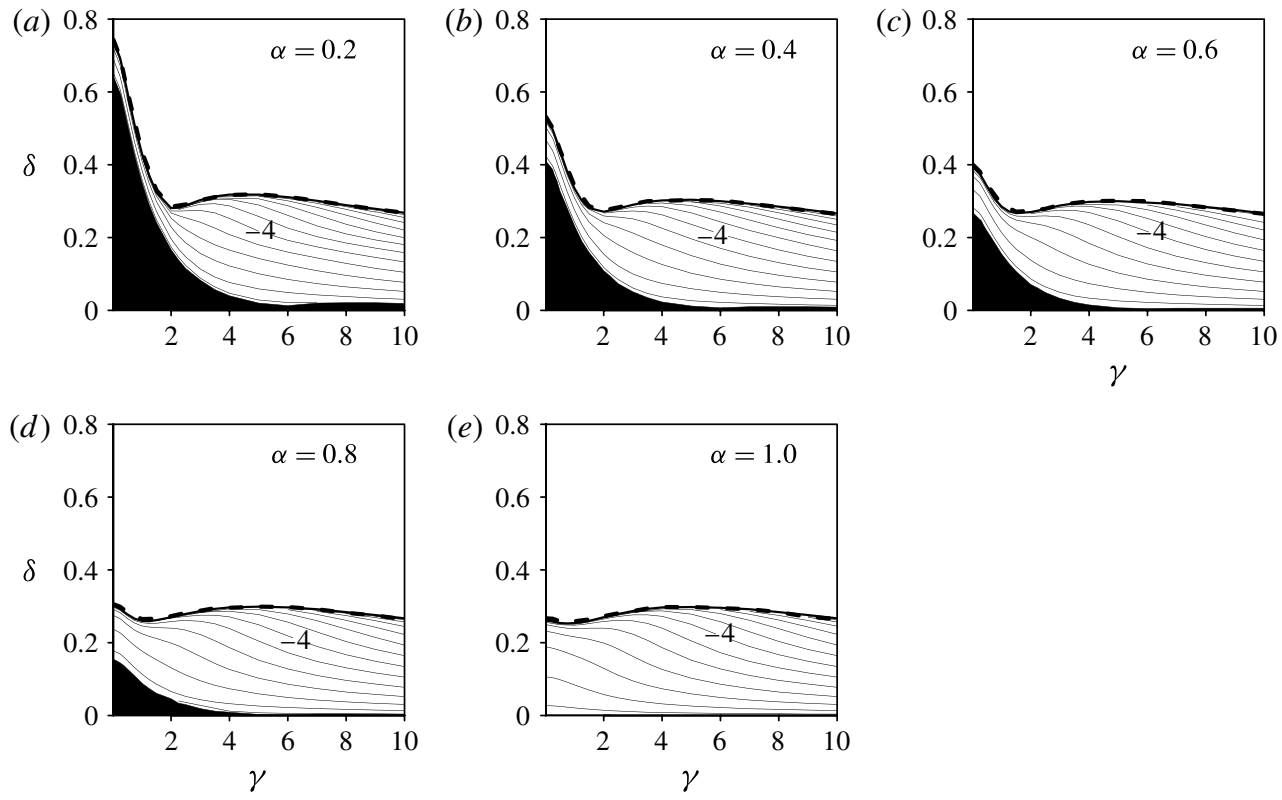

FIGURE 8. Logarithmically $\left(\log _{10}\right)$ scaled growth rates $\sigma_{r}$ of the most unstable mode in the $\gamma-\delta$ parameter plane. The contour interval is 0.5 . The bold line corresponds to when $\sigma_{r}=0$, while the dashed line corresponds to the boundary of nonlinear stability. Note that these two lines practically coincide.

of $J$ and $E$. We recover this result for our $\gamma \neq 0$ equilibria, pointing towards the possible existence of additional branches of families of equilibria (which are likely to be unstable, as we have found no evidence for them in our nonlinear simulations). As can be seen from figure 9 , we do not have $\sigma_{r}=\sigma_{i}=0$ exactly at $\delta=\delta_{c}$, but the falling values of the lower branch of $\sigma_{i}$ as $n_{p}$ increases indicates that this result is likely to be recovered for infinite resolution.

To summarize, doubly-connected equilibria having large $\gamma$ tend to be stable at smaller distances than those having small $\gamma$. This is most notable at small $\alpha$. Additionally, the boundary of stability becomes independent of $\alpha$ for large $\gamma$, especially for $\gamma \gtrsim 2$ and $\alpha>0.2$. Note that Makarov et al. (2012) found that twovortex heton equilibria (a heton is a vortex structure where the vortices are located in different layers) are less stable at large $\gamma$. However, these differences in the effects of $\gamma$ can be attributed to the fact that the dynamics of a two-layer quasi-geostrophic system are very different when the lower layer is of finite depth (it is infinite in QGSW), as pointed out and discussed by Polvani et al. (1989).

\section{Nonlinear evolution}

We next examine the nonlinear stability of the equilibria, and by finding their margin of nonlinear stability, we aim to relate it to that of the linear problem. We also endeavour to give an overview of the general features which characterize the evolution of marginally unstable states, and how these change with $\gamma$ and $\alpha$. We first present the numerical framework for examining the evolution of the states in $\S 4.1$ and then present our results in $\S 4.2$. 
(a)

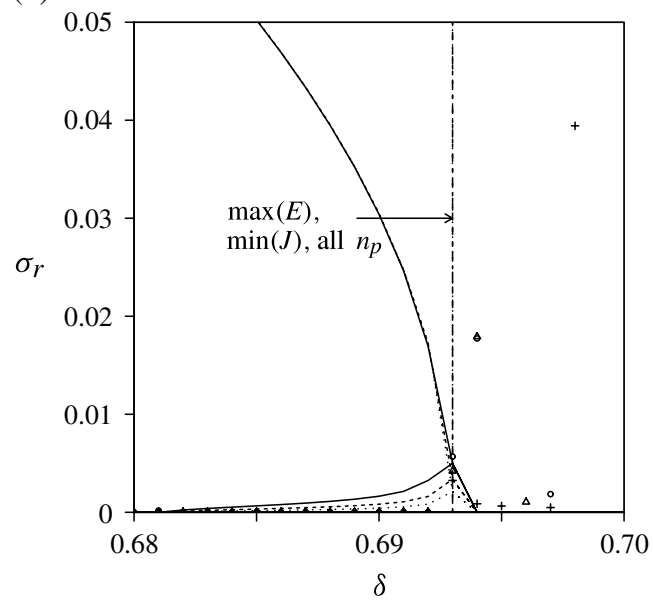

(b)

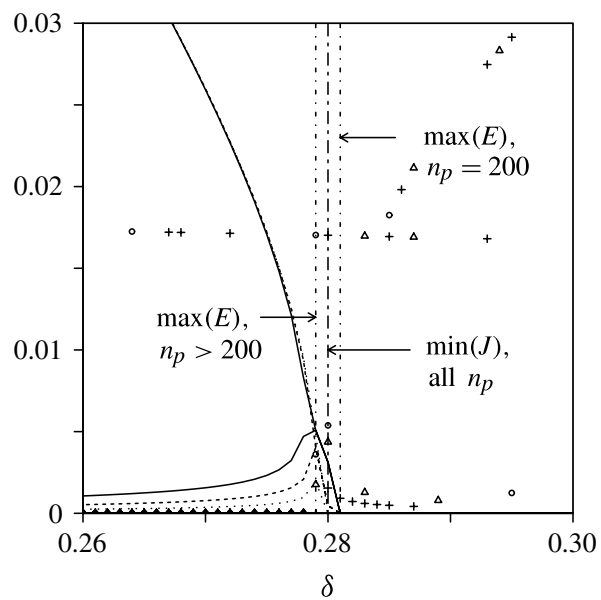

(c) $\quad\left(\times 10^{-5}\right)$

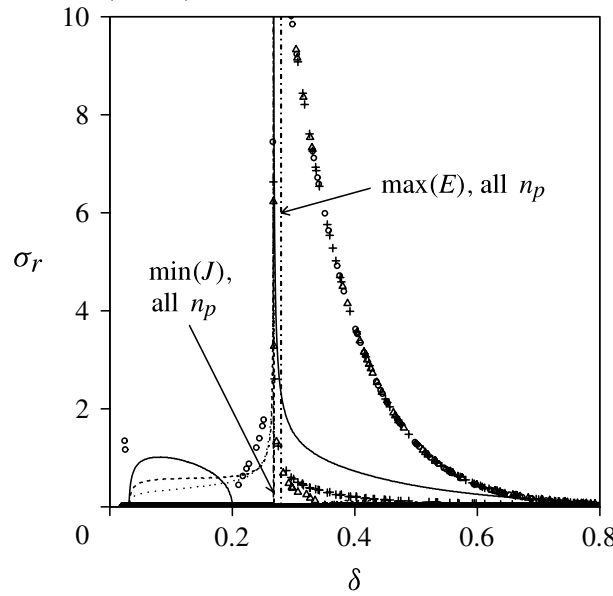

FIGURE 9. The growth rates (lines) and frequencies (points) of the five most unstable modes as a function of distance for $(a) \gamma=0.25$, (b) $\gamma=2$, and $(c) \gamma=10$ at $\alpha=0.2$. The different lines and symbols indicate different number of points $n_{p}$ used on half of the vortex boundary: $n_{p}=200$ (solid line; circles), $n_{p}=400$ (dashed line; triangles), and $n_{p}=800$ (dotted line; pluses). The dash-dotted line indicates the maximum of $E$ and the short-long-dashed line the minimum of $J$ for each of the cases, as marked in the figure. Note the different scale for $\sigma_{r}$ in each of the figures.

\subsection{Method}

We examine the nonlinear evolution of equilibria by exploiting the natural 'contour dynamics' formalism, as discussed in $\$ 2.1$. As we only need to keep track of the boundaries of the vortex-patch equilibria, we discretize the boundary of each vortex by a self-adapting number of nodes connected by local cubic splines, and numerically solve (2.3) using the 'contour surgery' algorithm (Dritschel 1988, 1989). The algorithm splits the singular modified Bessel function $\mathrm{K}_{0}$ appearing in (2.3) into a logarithmic part, $\log \left|\boldsymbol{x}-\boldsymbol{x}_{k}\right|$, which is integrated explicitly, and a non-singular remainder part, which is numerically integrated by two-point Gaussian quadrature. A 
fourth-order Runge-Kutta time integration method is used with a fixed, standard time step of $\Delta t=0.025$.

The number of nodes adjusts in response to dynamic changes in the contour curvature. We initially start with a slightly disturbed doubly-connected equilibrium, where each vortex patch is represented by an initial $n=n_{0}=400$ number of nodes. In order to avoid biasing the evolution towards any particular instability mode, we randomly perturb the $x$ and $y$ coordinates of each node by up to $1 \%$ of $\Delta \theta=2 \pi / n_{i}$, where $n_{i=1,2}=n_{0}$ is the initial number of nodes used to represent each contour. Throughout the evolution, the nodes are redistributed every 8 time steps, using a dimensionless node separation parameter $\mu=0.2$ and a large-scale length $L_{c}=P /\left(\mu n_{0}\right)$, where $P=P_{1}+P_{2}$ is the sum of the perimeters of the equilibrium contours. Contour surgery, which either splits a contour into two parts or joins two contours together, is performed with the same frequency whenever the distance between two contours or two parts of the same contour decreases below the 'cutoff' scale $d_{c}=\mu^{2} L_{c} / 4$. This allows the representation of many complex phenomena which typify vortex-patch dynamics, such as filamentation and splitting. The numerical details of the procedure, along with a full discussion of the now standard settings of the control parameters $\mu, d_{c}$ and the frequency of the surgery and node redistribution can be found in Fontane \& Dritschel (2009).

We consider five different area ratios $\alpha=0.2-1.0$ at $\Delta \alpha=0.2$, as well as $\gamma=0.02$, $0.25, \gamma=0.5-3.5$ at $\Delta \gamma=0.5$ and $\gamma=4-10$ at $\Delta \gamma=1$ (16 $\gamma$ cases in total). The small- $\gamma$ states are examined more closely as in this regime there is a steep decrease in the growth rates, as seen in figure 8. We evolve the equilibria for at least 200 particle rotation periods $T_{p}=2 \pi / \Omega_{p, 2}$ of the smaller vortex. One $T_{p}$ is equivalent to the amount of time it takes for a particle to complete a circuit of the vortex boundary. Based on the time scales which typify the oceans, this was deemed sufficiently long to assess nonlinear stability.

\subsection{Results}

In general, unlike the reverse of the statement, linear stability does not imply nonlinear stability. However, we find that the boundaries of linear and nonlinear stability coincide to within $\Delta \delta=0.008$, as shown by the dashed lines in figure 3 . The accuracy of this coincidence increases with $\gamma$. We expect that with increasing resolution, these discrepancies would reduce to zero.

The unstable evolution of vortices having $\delta<\delta_{c}$ near the margin of stability reveals a surprisingly rich set of behaviours. We follow Dritschel \& Waugh (1992), and classify them based on the change in the area of the two largest PV patches in the domain: $A_{1}^{r}=A_{1}^{f} / A_{1}^{i}$ and $A_{2}^{r}=A_{2}^{f} / A_{2}^{i}$, where the superscripts $i$ and $f$ indicate the initial and final states (the $A_{j}^{f}$ are calculated as a time-average once an instability has taken place). Four regimes have been identified, two of which are further divided into two categories. The first is the elastic interactions regime, in which the states do not change in area, i.e. $A_{1}^{r}=A_{2}^{r}=1$. Based on a visual examination of the evolution of the states, this regime may be further subdivided into stable states $\left(\mathrm{EI}_{s}\right)$, which do not change in shape, and vacillating states $\left(\mathrm{EI}_{v}\right)$. The vacillating states undergo a cycle of approaching and moving away from each other, adjusting their shapes as the distance between them changes, but not changing in area. The second regime is partial straining out (PSO), where the areas of either one $A_{1}^{r}=1,0<A_{2}^{r}<1\left(\mathrm{PSO}_{o}\right)$ or both $0<A_{1}^{r}<1,0<A_{2}^{r}<1\left(\mathrm{PSO}_{b}\right)$ of the vortices decreases. The third and fourth regimes are those where the larger vortex grows in size, by either partially destroying 
(a)

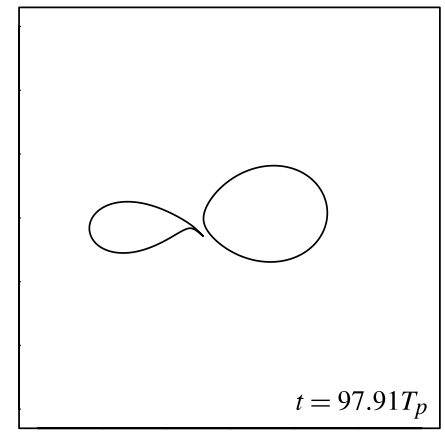

(d)

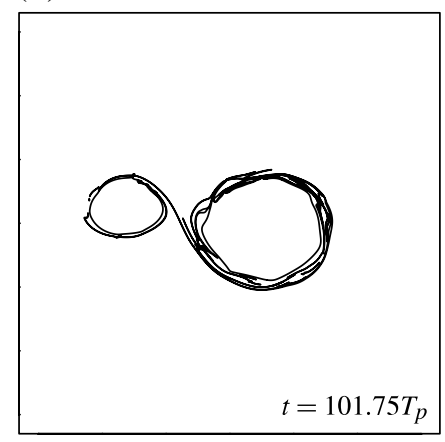

(b)

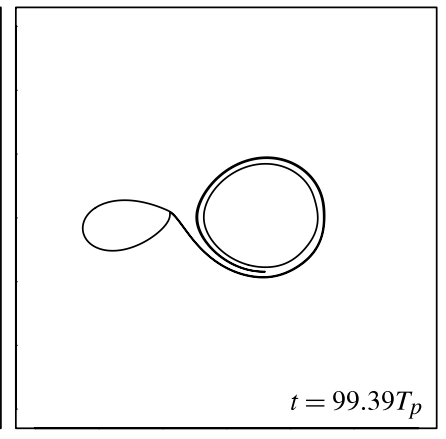

(e)

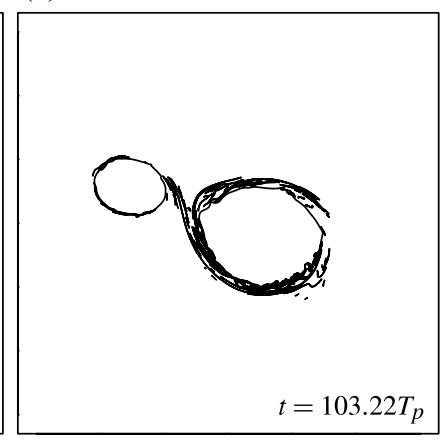

(c)

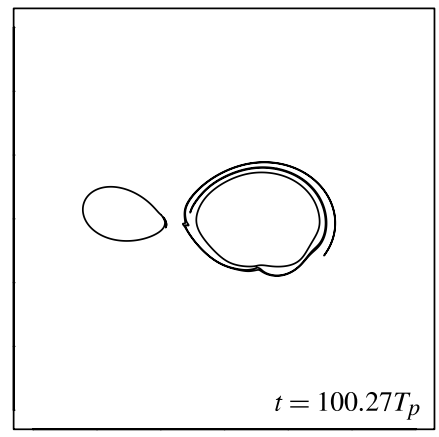

$(f)$

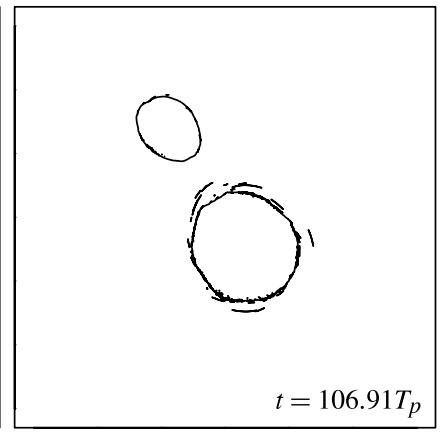

FIGURE 10. An example of the evolution of a state undergoing partial straining out $\mathrm{PSO}_{b}$. We show the case $\gamma=1, \alpha=0.4$ and $\delta=0.339$. The plotted domain has $|x|,|y| \leqslant 3.3$. See also supplementary movie 2 .

the smaller one $A_{1}^{r}>1,0<A_{2}^{r}<1$ in partial merger (PM) or wholly absorbing it in complete merger $A_{1}^{r}>1, A_{2}^{r}=0(\mathrm{CM})$.

Figure 10 shows an example of the $\mathrm{PSO}_{b}$ regime, with $\gamma=1, \alpha=0.4$ and $\delta=0.339$. (Note that figures $10-13$ are complemented by supplementary movies $2-5$.) The smaller vortex $\left(\mathrm{V}_{2}\right)$ undergoes a wave-3 disturbance and sheds a filament which wraps around the larger vortex $\left(\mathrm{V}_{1}\right)$. Fine-scale structures develop on the boundary of $\mathrm{V}_{1}$, which then get partly transferred also onto the boundary of $\mathrm{V}_{2}$. Figure 14 shows how the area of $\mathrm{V}_{1}$ (bold line), $\mathrm{V}_{2}$ (thin line), and their sum (dashed line) changes with time. We see that soon after the onset of instability both of the vortices reach constant areas which do not change, even though some small-scale structures still exist on their boundaries (this is shown in figure $10 f$ ). In the case shown in figure 10, the small-scale filamentary debris become so small that they eventually get dissipated away, and we are left with two asymmetric vortices. However, the filaments may also roll up to form small satellite vortices (much smaller than the two dominant vortices). Additionally, the filament shed by $\mathrm{V}_{2}$ is not always transferred back onto the boundary of $\mathrm{V}_{2}$. The $\mathrm{PSO}_{o}$ regime is qualitatively similar to $\mathrm{PSO}_{b}$, but $\mathrm{V}_{1}$ does not increase in area. However, as can be seen from the plots in figure 14 corresponding to the $\mathrm{PSO}_{o}$ and $\mathrm{PSO}_{b}$ regimes, the decrease in area of $\mathrm{V}_{1}$ is not large. In fact, at most it decreases by $2.5 \%$ (we say a state falls into the $\mathrm{PSO}_{b}$ regime if the area of $\mathrm{V}_{1}$ changes by more than $1 \%$ ). 
(a)

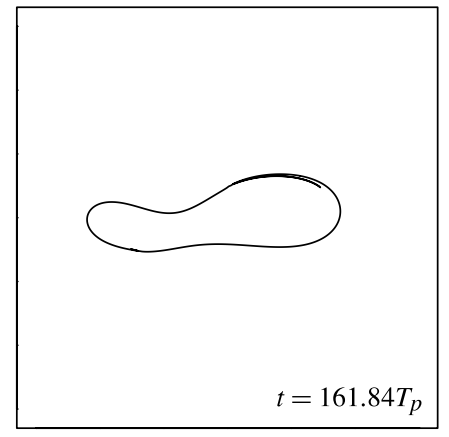

(d)

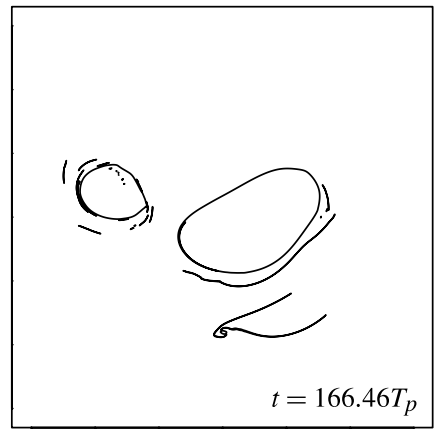

(g)

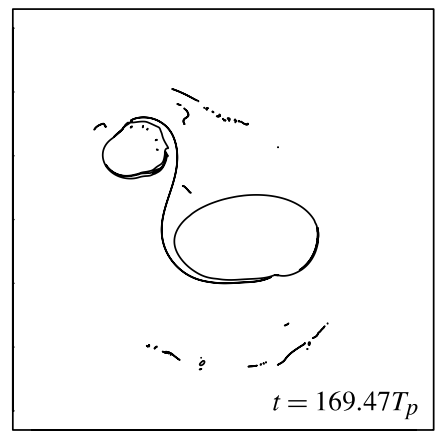

(b)

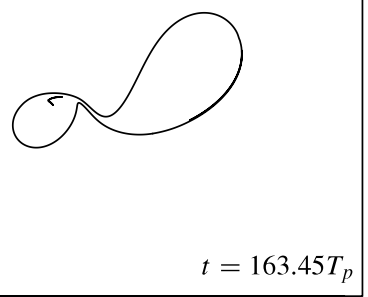

(e)

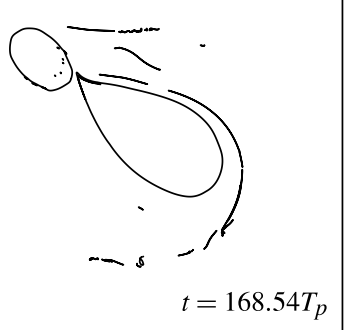

(h)

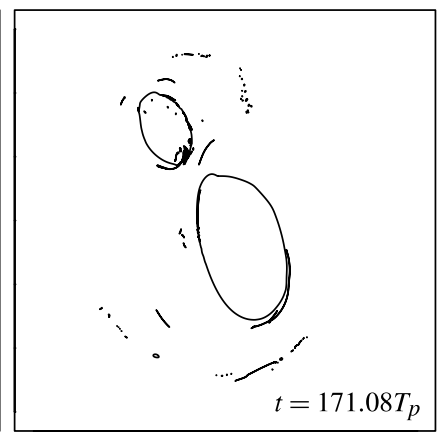

(c)

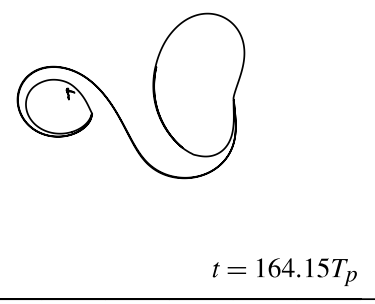

(f)

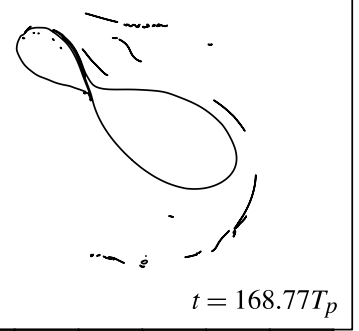

(i)

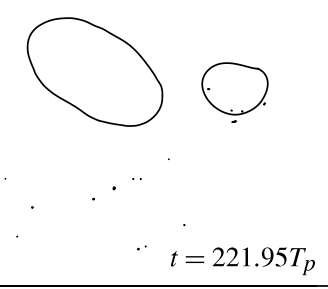

FIGURE 11. An example of the evolution of a state undergoing partial merger PM. We show the case $\gamma=2, \alpha=0.6$ and $\delta=0.270$. Here, $|x|,|y| \leqslant 3.3$. See also supplementary movie 3 .

Figure 11 shows an example of the partial merger regime, with $\gamma=2, \alpha=0.6$ and $\delta=0.270$. The vortices merge, but after only $\sim 3 T_{p}$ the resulting vortex splits, shedding some filamentary debris. After another $5 T_{p}$ it reconnects for $2 T_{p}$ before splitting again and shedding more debris. As can be seen from the PM plot of figure $14(c)$, the size of $\mathrm{V}_{1}$ does not change after the second split, unlike that of $\mathrm{V}_{2}$, which sheds a few small filaments, until it too reaches a size which does not change (shown at $t=221.95 T_{p}$ in figure $11 i$ ). The final area ratio between the two vortices gets halved to $\alpha^{f} \approx 0.27$, and despite the shedding of filaments during the splitting process, only $\sim 2.4 \%$ of the total area of the two vortices is lost. This series of merging and splitting events, sometimes occurring multiple times, is typical of the PM regime. The amount of area lost to filaments varies with $\gamma$, and is maximum at $26.4 \%$ 
(a)

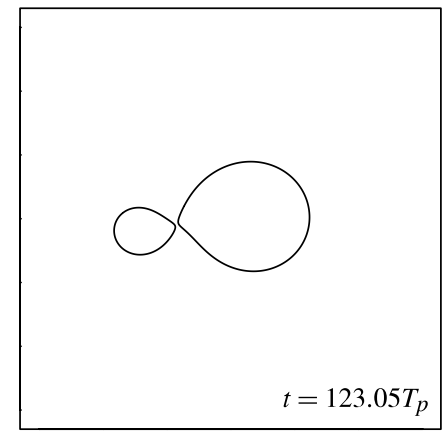

(d)

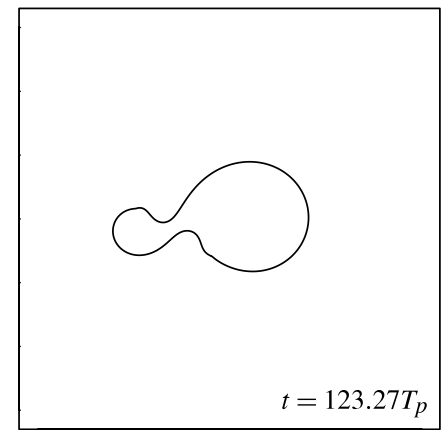

(b)

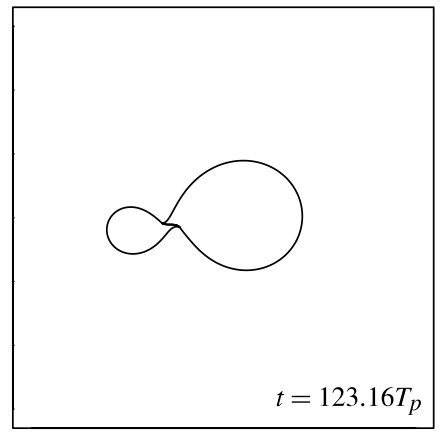

(e)

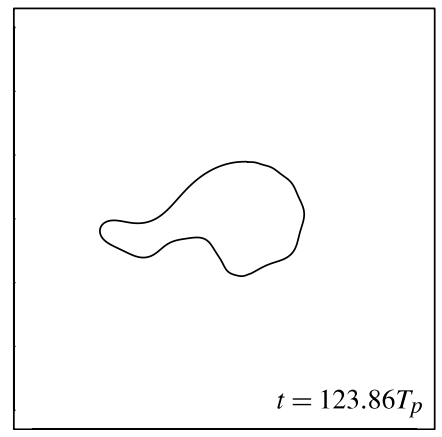

(c)

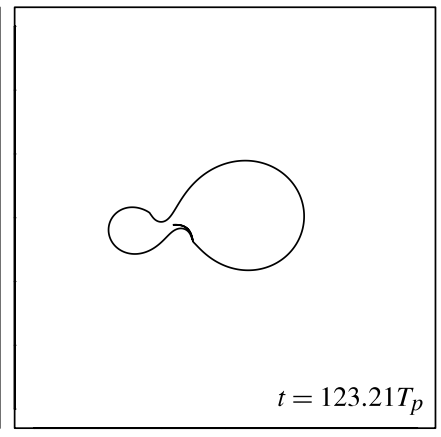

$(f)$

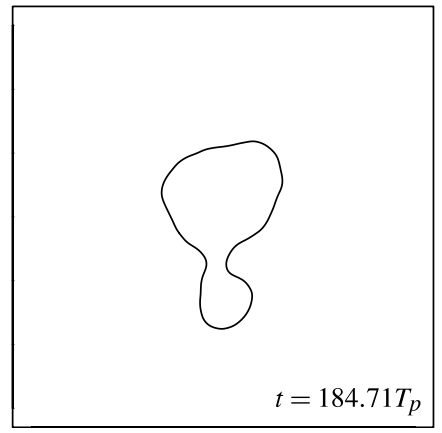

FIGURE 12. An example of the evolution of a state having large $\gamma$ which undergoes complete merger CM. We show the case $\gamma=10, \alpha=0.2$ and $\delta=0.268$. Here, $|x|,|y| \leqslant 3.3$. See also supplementary movie 4 .

for $\gamma=0.25, \alpha=1.0$ and $\delta=0.266$, where there is considerable filamentation and interaction of the filaments with the boundaries of both $\mathrm{V}_{1}$ and $\mathrm{V}_{2}$. For unstable equilibria with $\gamma>1$, no more than $5 \%$ of the total area is lost, and states having $\gamma=4$ (the largest $\gamma$ in the PM regime) have the smallest area loss of $\ll 1 \%$, despite repeating the merging and splitting process up to five times before reaching a quasiequilibrium state. For the large- $\gamma$ cases, it appears that when the state merges, it attempts to reach a quasi-steady simply-connected state by shedding small filaments, but it is not able to do so and eventually splits. Note that in realistic geophysical flows, in which vortices are often subjected to a straining flow from e.g. the presence of other vortices in their proximity, the effects of 'vortex stripping' (vorticity being torn away from the vortex periphery) and diffusion (if additionally viscous effects were present) would affect the evolutionary path of unstable vortices (Legras \& Dritschel 1993; Mariotti, Legras \& Dritschel 1994). This might be expected to affect both the PSO and PM regimes of instability, as the strain would prevent filaments from rolling up to form smaller vortices.

When merger occurs at large $\gamma$, large-amplitude waves obeying the modified Korteweg-de Vries (KdV) equation to leading order in $L_{D}$ (see Nycander et al. 1993) propagate on the vortex boundary, and are responsible for the splitting of the vortex. As $\gamma$ increases the splitting no longer takes place, or, as in the cases of $\gamma \lesssim 5$ having $\alpha \geqslant 0.8$ and $4 \lesssim \gamma \lesssim 6$ having $\alpha \lesssim 0.4$, a near-instantaneous split lasting much less than one $T_{p}$ occurs and the vortices immediately reconnect. In figure 12 we see an example of a case having $\gamma=10, \alpha=0.2$ and $\delta=0.268$ which 
(a)

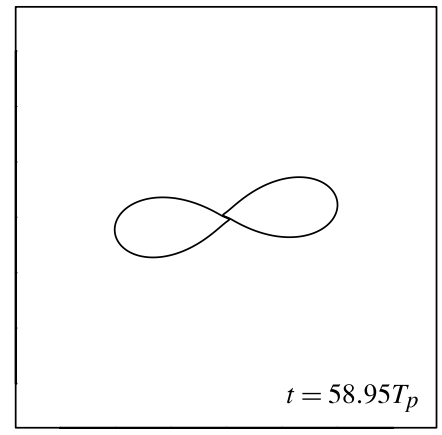

(d)

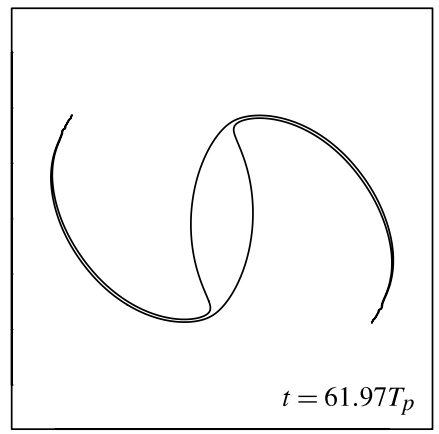

(g)

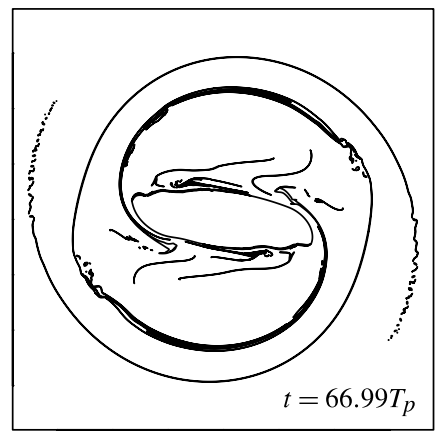

(b)

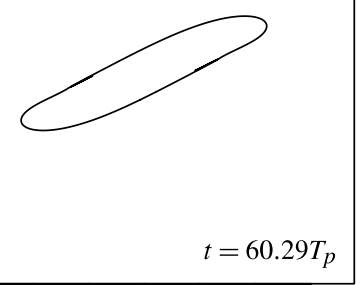

(e)

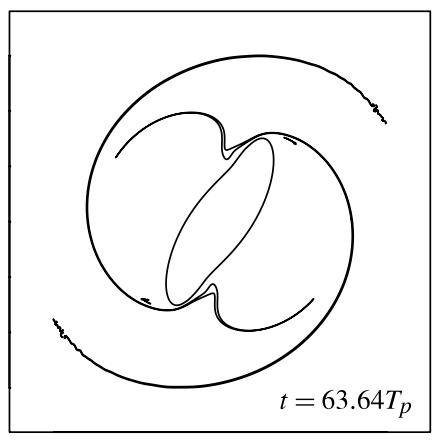

(h)

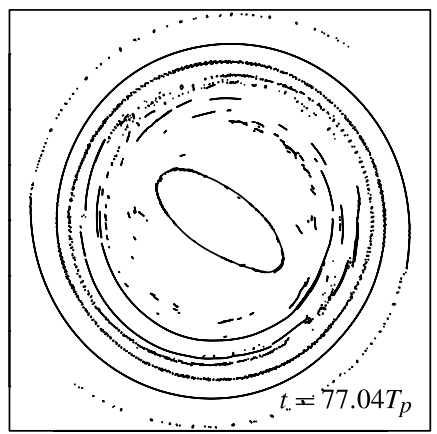

(c)

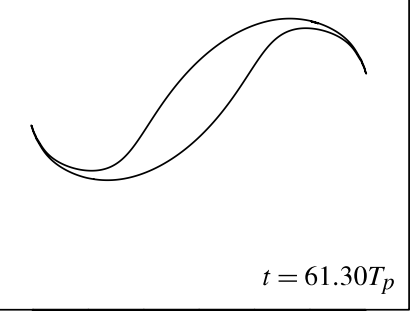

(f)

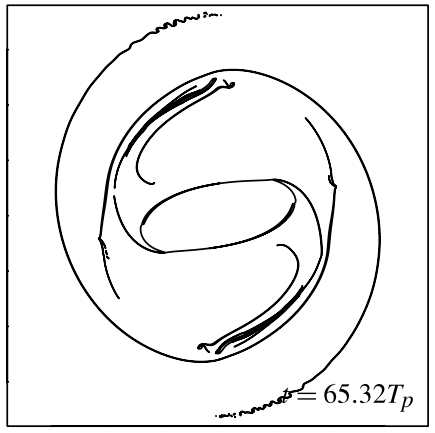

(i)

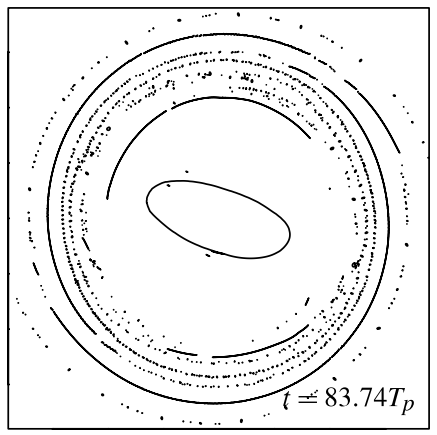

FIGURE 13. An example of the evolution of a state having small $\gamma$ which undergoes complete merger CM. We show the case $\gamma=0.02, \alpha=1.0$ and $\delta=0.266$. Here, $|x|,|y| \leqslant 3.8$. See also supplementary movie 5 .

undergoes complete merger. The waves propagating slowly along the merged boundary are obvious immediately after merger. Note that although the merged state is only quasi-steady, it nevertheless periodically repeats regular shapes, as shown at times $t=123.27 T_{p}$ and $t=184.71 T_{p}$ in figure $12(d, f)$. The asymmetry of the recurring dumbbell-shape is dependent on the initial $\alpha$ of the doubly-connected state: as $\alpha$ grows, the dumbbell becomes increasingly symmetric. As can be seen from the dashed line in figure $14(d)$, essentially no area is lost to filamentation during or after merger. Apart from special cases having $\gamma=0.02$ and $\alpha=1.0$, the CM regime occurs only for large $-\gamma$ states having $\gamma \geqslant 2$. States having small $\alpha \leqslant 0.4$ and $\gamma \leqslant 4$ produce the most 

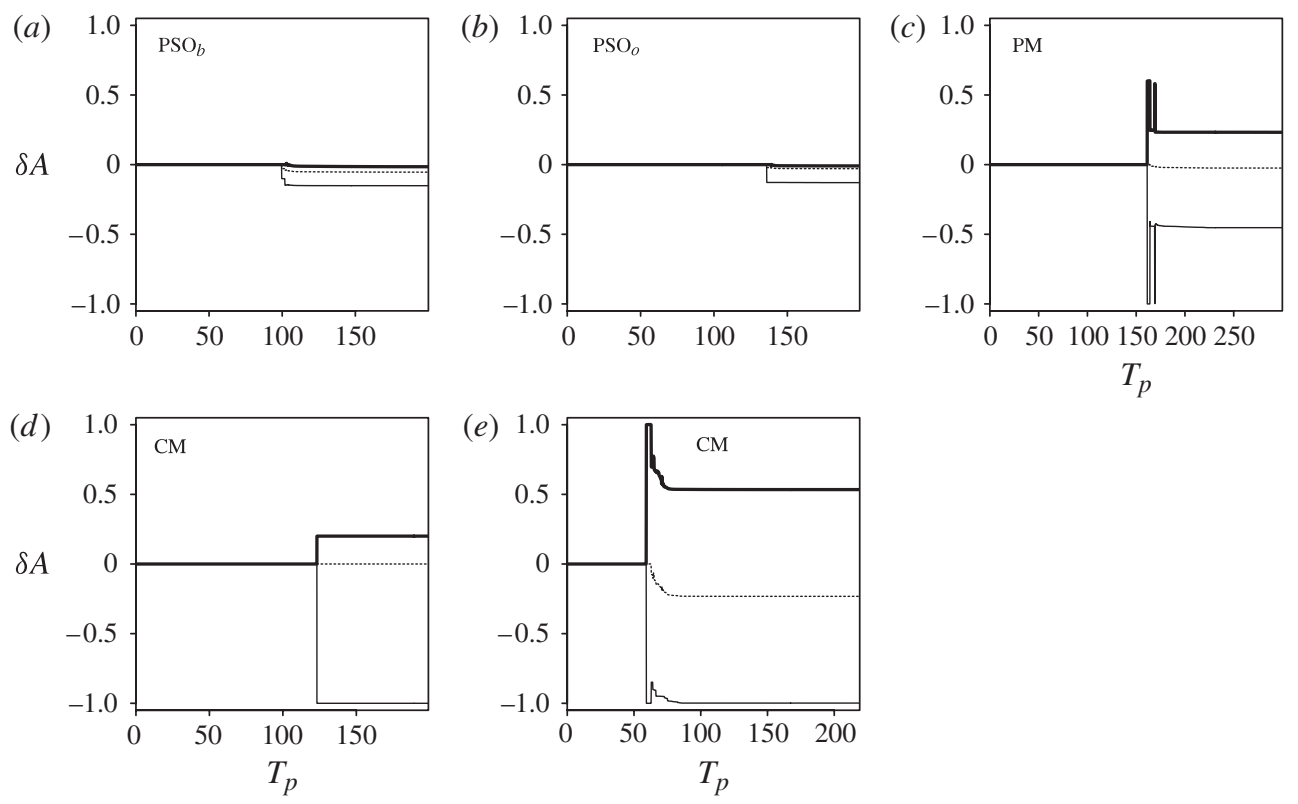

FIgURE 14. The fractional change in area $\delta A=A / A^{i}-1$ of the vortex with largest area (bold line), second largest area (thin line), and in the total area of the two largest patches of vorticity (dotted line). We see the cases corresponding to figures 10-13, i.e. (a) $\gamma=1$, $\alpha=0.4, \delta=0.339$ for $\mathrm{PSO}_{b}$; (b) $\gamma=2, \alpha=0.2, \delta=0.285$ for $\mathrm{PSO}_{o}$; (c) $\gamma=2, \alpha=0.6$, $\delta=0.270$ for PM; and $(d) \gamma=10, \alpha=0.2, \delta=0.268$ and $(e) \gamma=0.02, \alpha=1.0, \delta=0.266$, for CM.

filamentation, losing $2.7 \%$ of the total area. The behaviour of these states is similar to those in the PM regime, where merged simply-connected states shed PV while trying to recover a quasi-steady state. In the CM regime, such a quasi-steady state is reached quickly, and no further splitting occurs. States having large $\alpha$, and larger $\gamma$ shed essentially no PV during or after merger.

As mentioned above, cases having $\gamma=0.02$ and $\alpha=1.0$ exhibit atypical behaviour for the small- $\gamma$ regime, as shown for $\delta=0.266$ in figure 13. The state merges, and then by a wave- 4 disturbance sheds filaments from both tips (in general, such a state loses $\sim 23 \%$ of its total area, see figure 14e). These filaments then interact with the vortex boundary in a very complex way, until a quasi-steady elliptical vortex surrounded by a sea of filamentary debris is formed. These filaments tend not to roll up into small vortices, unlike what is sometimes seen in the $\mathrm{PSO}_{o}$ and $\mathrm{PSO}_{b}$ regimes for sufficiently thick filaments (for an explanation of this behaviour, see Waugh \& Dritschel 1991). This type of evolution has been previously described in the barotropic case by Dritschel (1995), who showed that even slight departures from the initially symmetric state, e.g. $\alpha=0.99$, causes the vortex to instead exhibit a wave-3 instability after merger. We obtain similar results for $\gamma=0.02$. However, for vortices with $\gamma \gtrsim 0.5$ we find that such a small departure from symmetry does not affect the nature of the nonlinear evolution. Note that we do not find complete merger for any other cases with $0.02<\gamma<2$.

An overview of the simulations carried out for states with $\alpha=0.4$ is shown in figure 15. As can be seen from the figure (the other $\alpha$ examined produce similarly 
(a)

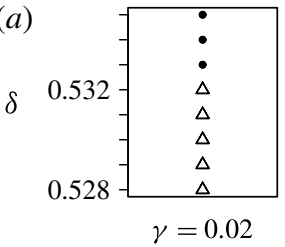

(d)
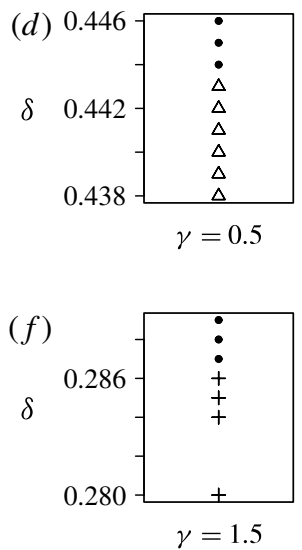

(b)

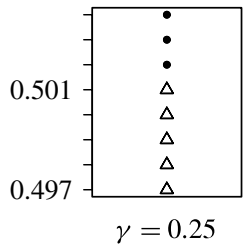

(e)

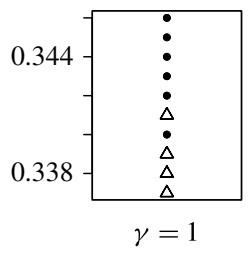

$(g)$

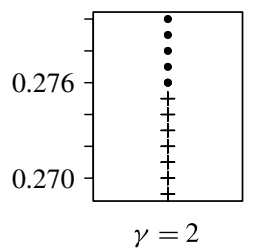

(c)

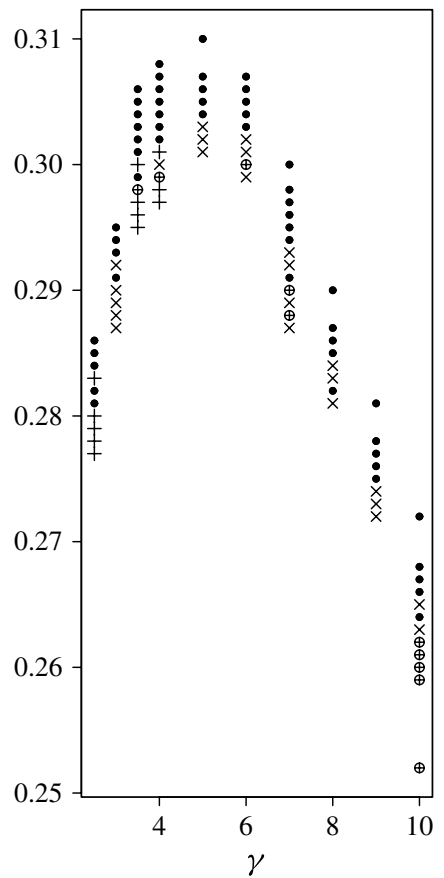

FIGURE 15. An overview of the simulations carried out for states with $\alpha=0.4$. The symbols denote the following: $\bullet$, stable state; $\oplus$, vacillations; $\bigcirc, \mathrm{PSO}_{o} ; \triangle, \mathrm{PSO}_{b} ;+, \mathrm{PM}$; and $\times, \mathrm{CM}$.

complex results), it is very difficult to divide up the parameter space based on the type of instability it experiences. However, below we attempt to do so, also tying this in with the results of the linear stability analysis. For $\alpha=0.2-0.6$ at small $\gamma \lesssim 0.5-2$ there are only PSO evolutions (the lower $\gamma$ values correspond to higher $\alpha$ ). The location of this boundary roughly corresponds to the boundary between the steep and flat contours in figure 8. The PSO regime is absent for $\alpha \geqslant 0.8$, where instead the small $\gamma \lesssim 2$ states fall into the PM regime. For $\alpha=0.2-0.6$ the PM regime falls between $1 \lesssim \gamma \lesssim 4$ (again, the lower $\gamma$ correspond to higher $\alpha$ ). The PM regime corresponds to the region of the $\sigma_{r}$ diagrams where there is a transition from the small- $\gamma$ to large- $\gamma$ instability modes. Although larger- $\alpha$ states do not have a region of steep $\sigma_{r}$ contours nor the PSO regimes of evolution, the transition region is still present, only for smaller $\gamma$ than in the lower- $\alpha$ states. The region of flat $\sigma_{r}$ contours at large $\gamma$ corresponds to the $\mathrm{CM}$ regime for all $\alpha$. Note that both at the boundary of the PM and CM regimes, and in the CM regime itself, we also have vacillations (see supplementary movie 6 for an example of this).

Lastly, we find that the final vortex area ratio $\alpha^{f}$ is always less than the initial one $\alpha^{i}$ (henceforth we omit the superscript $i$ for ease of notation). This is shown in figure 16, which shows $\alpha^{f}$ for each $\delta$ considered and for the five different $\alpha$ examined. The area of $\mathrm{V}_{2}$ never increases, but that of $\mathrm{V}_{1}$ does for all $\alpha$. For low $\alpha \lesssim 0.6$ the highest $\alpha^{f}$ are obtained in the PSO regime; on the other hand for $\alpha \gtrsim 0.6$, this regime of evolution is absent. Note that for the barotropic case, Dritschel (1995) has also found that $\mathrm{V}_{2}$ never increased its size, and that $\mathrm{V}_{1}$ did, but only for $\alpha \gtrsim 0.6$. Additionally, when $\gamma=0, \alpha^{f}<0.5$ always, which for $\gamma \neq 0$ changes to $\alpha^{f}<0.6$. 


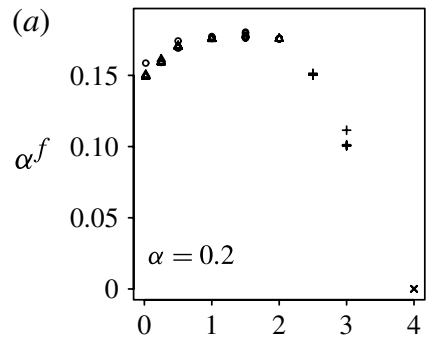

(b)

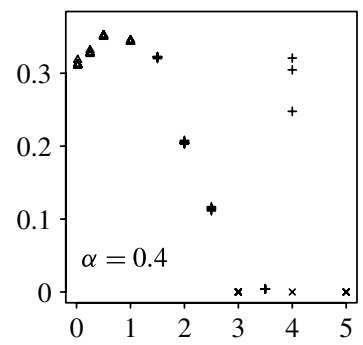

(d)

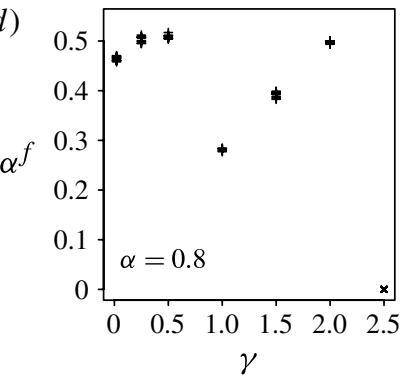

(e)

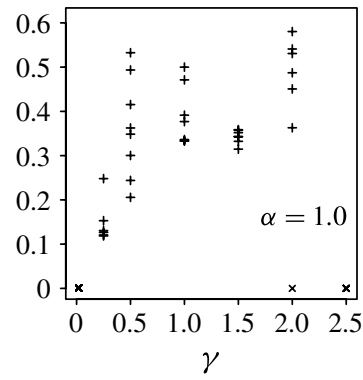

(c)

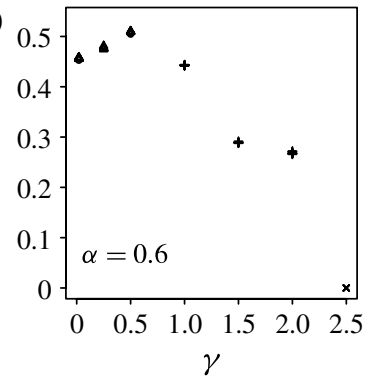

FIGURE 16. $\alpha^{f}$ as a function of $\gamma$ for the unstable cases having different initial area ratios. The symbols denote the following: $\mathrm{O}, \mathrm{PSO}_{o} ; \triangle, \mathrm{PSO}_{b} ;+, \mathrm{PM}$; and $\times, \mathrm{CM}$. Note that $\gamma$ greater than the ones shown in each of the figures have only instabilities in the CM regime.

\section{Transitions between simply- and doubly-connected equilibria}

In a previous paper, the stability of two-fold symmetric simply-connected (SC) vortex-patch equilibria was examined (Płotka \& Dritschel 2012). The families of these dumbbell-shaped equilibria are fully spanned by $\gamma$ and their aspect ratio $\lambda$, which is the ratio of the minor to major axes of the vortex patch. Similarly to the doublyconnected (DC) states, their limiting state consists of two vortices joined at a single point. The instability types of an SC state range from the vortex shedding a single filament - which as $\gamma$ is increased may roll up to form a smaller vortex - to a split into two symmetric vortices. This means that a pair of quasi-steady co-rotating vortices with area ratio $0<\alpha \leqslant 1$ is formed. Conversely, as was seen above, during instability many DC states, especially those with larger $\gamma$, often merge into a single quasi-steady vortex, with either no, or negligible, filamentation. Through merging and splitting, they may also undergo a transition from one DC state to another quasi-steady DC state.

Cerretelli \& Williamson (2003b) studied families of barotropic equilibria, starting from a DC state and transitioning into an SC one. Experimental studies (Meunier \& Leweke 2001; Cerretelli \& Williamson 2003a) have shown that prior to merger, symmetric barotropic vortices may diffusively merge into a dumbbell-shaped vortex. Below we examine both the inviscid and dissipative transitions between DC and SC states, and between two DC states.

Dritschel $(1985,1986)$ examined transitions between barotropic SC and DC states having identical circulation, angular impulse, centroid location, energy, area and vorticity. Waugh (1992) performed a similar analysis for transitions between a single and two barotropic elliptical vortices. They found that such transitions are possible, even if they occur between two unstable states, and thus the resulting states are short-lived. 
We say that a transition between two equilibrium states is complete if the quantities mentioned above are conserved. The statement of their conservation is equivalent to the conservation of $J, E$ and area (for fixed $\gamma$ ). By finding two (or more) states with identical $(J, E)$ at a fixed $\gamma$, we identify complete transitions between SC and DC states, and between two DC states (note that we keep the total area $A=\pi$ in all cases). We concentrate on complete transitions from an unstable to a stable state, as these are the most likely to occur and last long times, even though complete transitions between two stable and two unstable states also exist. Based on the results found here and in Płotka \& Dritschel (2012), many transitions between distinct equilibrium states appear possible. Numerous instances have been found where vortices merge or split with virtually no filamentation or loss of area, especially at large $\gamma$, thus conserving total area, $J$ and $E$.

We first examine the effects of non-zero $\gamma$ on complete transitions between SC and DC equilibria. For a complete transition to be possible, the angular impulse and energy of an SC state must be equal to that of a DC state, i.e. $(J, E)_{S C} \equiv(J, E)_{D C}$. Figure 17 shows $J$ (thin line) and $E$ (dashed line) of DC states in the $\alpha-\delta$ parameter plane for $\gamma=0.25,1,3$ and 10 . The contour levels shown correspond to $(J, E)_{S C}$ at the same $\gamma$ and for aspect ratios $0<\lambda<1$, with $\Delta \lambda=0.010$. The intersection points of a pair of contours associated with the same $\lambda$ (for illustration, marked by a black dot on one contour pair in the figure) correspond to a possible complete transition between two states. To determine a range of possible complete transitions, at a fixed $\gamma$ we look at contour levels corresponding to SC states with a finer division $\Delta \lambda=0.001$, and locate intersection points of $(J, E)$ in the $\alpha-\delta$ parameter plane. Figure 18 shows a summary of these complete transitions for $\alpha$ (top row) and $\delta$ (bottom row) as a function of $\lambda$. We find that complete transitions are possible between two stable, two unstable, and an unstable SC and stable DC state, as indicated by the different symbols in the figure.

We see that for $\gamma \lesssim 1$, complete transitions are possible between a stable or unstable DC state and an unstable SC state. These complete transitions are not unique, and for SC states with larger $\lambda$, both a stable and unstable DC state with the same energetics exists. The difference in $\alpha$ of the two possible DC states is small, as opposed to the difference in the distance between each of the vortices in the corresponding states. In contrast, for $\gamma>1$, only complete transitions between stable SC and DC states are possible, and these complete transitions are unique.

We next examine complete transitions between two DC states, denoted $a$ and $b$, so that $(J, E)_{D C}^{a} \equiv(J, E)_{D C}^{b}$. Figure 19 shows $J$ and $E$ of a $D^{b}$ state in the $\alpha-\delta$ parameter plane. The contour levels shown correspond to $(J, E)_{D C}^{a}$ at a fixed $\gamma$ and $(\alpha, \delta)$ with $\alpha$ in the range $0.1-1$ at $\Delta \alpha=0.1$ and $\delta=0.500$. Similarly as for complete transitions between SC and DC states, given $(\alpha, \delta)^{a}$ of $\mathrm{DC}^{a}$, we find the corresponding $(\alpha, \delta)^{b}$ of $\mathrm{DC}^{b}$ by looking for intersection points between the $J$ and $E$ contours (for illustration, these are marked by the black dots in the figure for a given contour level). We find that complete transitions between two stable, two unstable, and an unstable and stable state are possible. However, as complete transitions between two stable states are unlikely to occur in real flows, and complete transitions between two unstable states would be short-lived, we only present results for complete transitions between unstable and stable states. Figure 20 shows these complete transitions in the $\alpha-\delta$ parameter plane. Possible complete transitions are connected by a grey line. Note that states below the bold dashed line are unstable, and those above are stable.

From figure 20 we see that states with small $\gamma$, and also ones with small $\alpha$ regardless of $\gamma$, undergo complete transitions in which $\alpha$ remains unchanged, and only $\delta$ changes. As $\gamma$ is increased, complete transitions which change both $\alpha$ and $\delta$ are 
(a)

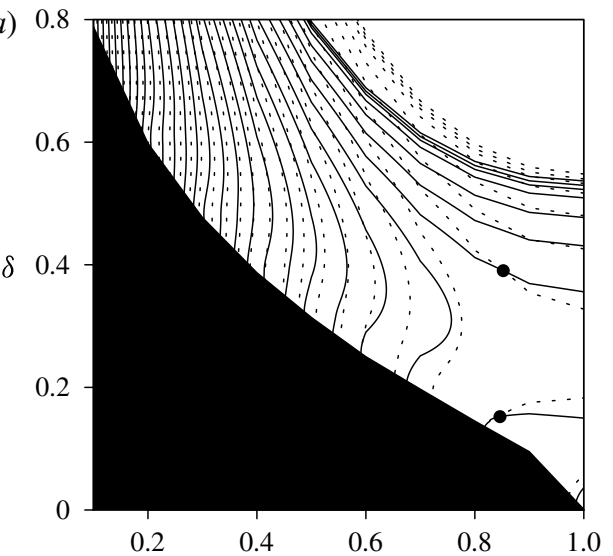

(c)

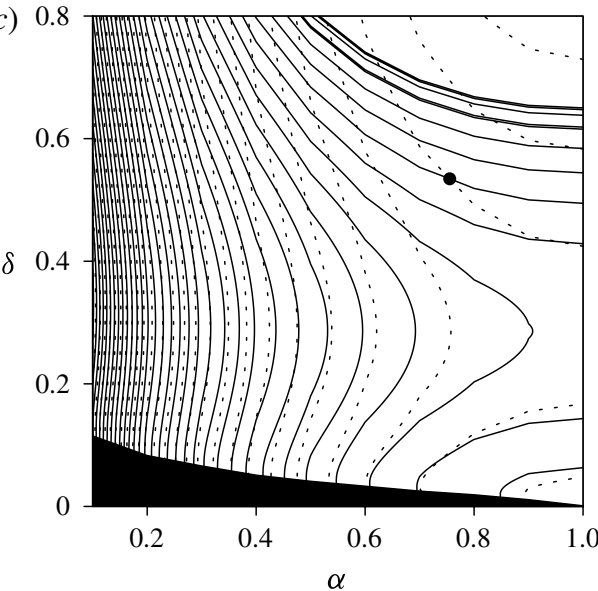

(b)

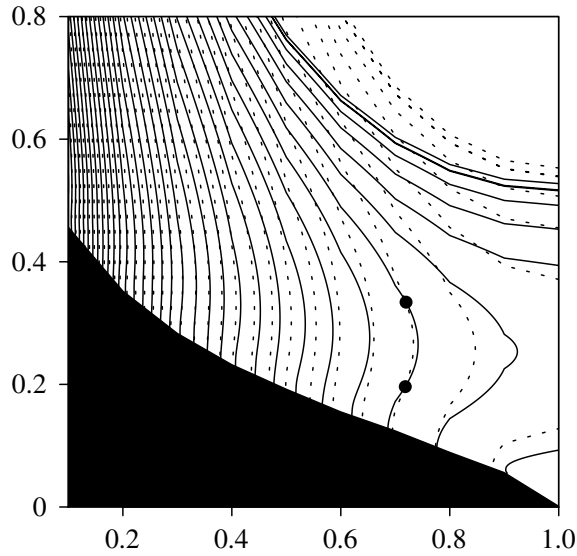

(d)

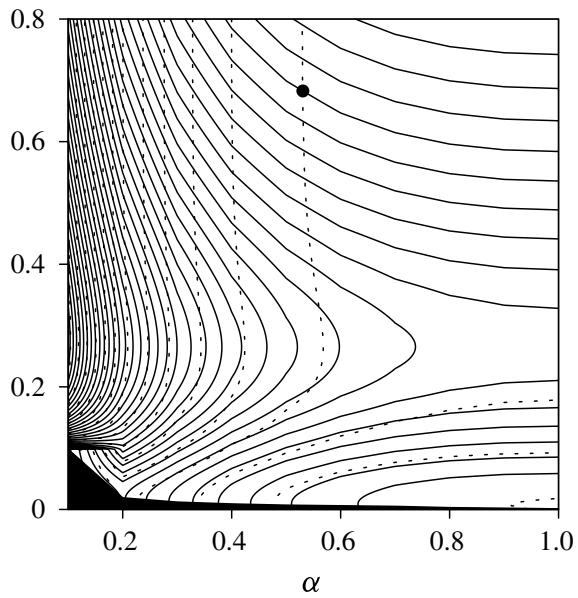

FIGURE 17. $J$ (thin line) and $E$ (dashed line) of DC states in the $\alpha-\delta$ parameter plane. We show $(a) \gamma=0.25,(b) \gamma=1,(c) \gamma=3$ and $(d) \gamma=10$. The contours extracted correspond to states with $\lambda=0-1$ at $\Delta \lambda=0.010$. The black dots mark the intersection points between the $J$ and $E$ contours corresponding to the SC state with the same $\lambda$, marking a possible complete transition from a pre-selected SC state. A complete transition into more than one unique DC state is possible when multiple black dots (i.e. multiple intersections) are present.

possible for increasingly small $\alpha$. In fact, when the maximum change in $\alpha$ is examined at each $\gamma$, we find that it increases almost perfectly linearly with $\gamma$, having a slope close to 0.057 . At $\gamma=0.25$ this maximum difference is 0.044 , while at $\gamma=10$ it is 0.591. Additionally, we see that for $\gamma \lesssim 1$ the change in $\delta$ between states is fairly uniform especially at a fixed $\alpha$ (with the standard deviation from the average being around 0.15 ), while for $\gamma>1$ the complete transitions are more varied, with complete transitions having both small and large changes in $\delta$ (here, the standard deviation is around 0.21). A final point to note is that for larger $\gamma$, there exist more possible complete transitions than for smaller $\gamma$.

As shown above, inviscid (complete) transitions from one equilibrium state to another are possible. However, they do not appear to be preferred. We have induced small perturbations (as in $\S 4$ ) on unstable equilibria for which inviscid transitions to a stable equilibrium are known to exist to see if such a predicted complete transition 
(a)
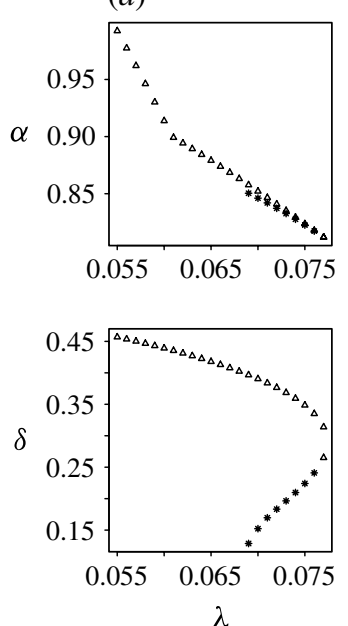

(b)
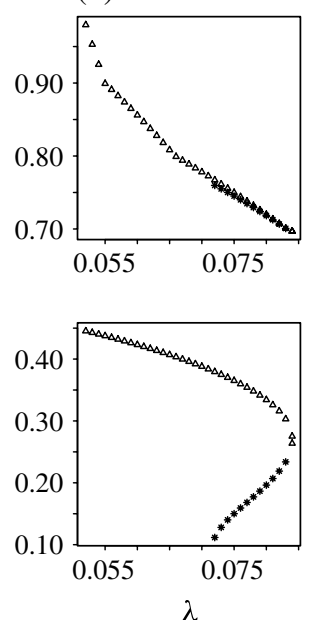

(c)
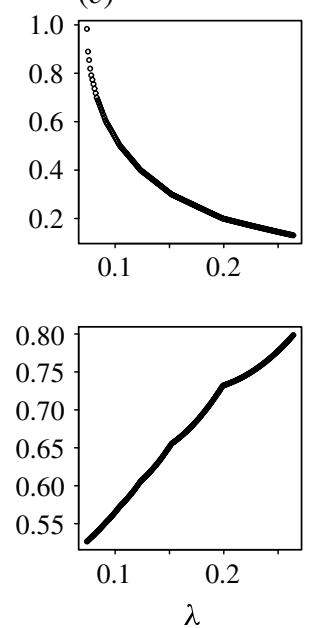

$(d)$
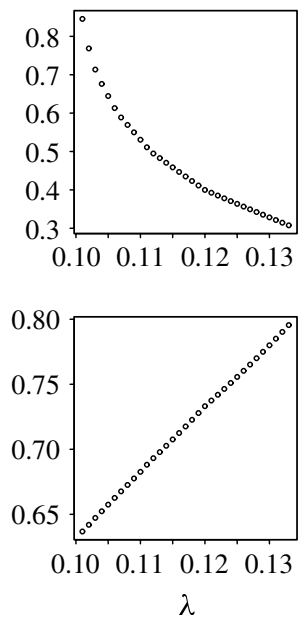

FIGURE 18. A summary of complete transitions possible between SC and DC states. In columns $(a-d)$ we show $\gamma=0.25,1,3$ and 10, respectively. The symbols indicate the stability regimes of (SC, DC): $\bigcirc$, (stable, stable); $\triangle$, (unstable, stable); and $*$, (unstable, unstable).

would occur. We find that for transitions from unstable SC states to stable DC states there is always loss of $J$ and $E$ (recall that such complete transitions are only possible for small- $\gamma$ states and note that we do not find complete transitions between an unstable DC state and a stable SC state, cf. figure 18). The same is true for transitions between two DC states at both small $\gamma$, and at large $\gamma$ for small $\alpha \lesssim 0.4$. At large $\gamma$ and large $\alpha \gtrsim 0.4$, we find that $J$ and $E$ are conserved, but rather than undergoing the complete transition into a DC state predicted by the above analysis, the state either exhibits vacillations around a stable steady state or complete merger, and thus transitions to a SC state. At large $\gamma$, all of the complete transitions between SC and DC states have been found to be between two stable states. The newly formed SC state has waves travelling on its boundary, and thus cannot be considered to be in equilibrium, as discussed below. This in turn implies that even at large $\gamma$ and large $\alpha$, inviscid transitions do not occur.

Although generally we do not find evidence for inviscid transitions, we observe that near the boundary of stability both small- and large- $\gamma$ states which undergo unstable evolution reach unsteady end states with waves travelling on their boundary, as seen in the previous section. The long-time persistence of these newly formed states points towards their oscillation around an underlying equilibrium state, albeit with energetics different from those of the initial steady state. However, determining the underlying equilibrium from energetics is not possible, as the waves, which occur on the vortex boundary where most of the kinetic energy of the state is present, carry (negative) energy. This makes the total energy of the system lower than that of the underlying equilibrium state. Damping the waves during the unstable evolution, while at the same time preserving area in order to be able to find the underlying equilibrium could be done, but is ad hoc. Additionally, as the waves also carry $J$, determining $\delta$ or $\lambda$ of the newly formed state (even from time-averaged values) is not meaningful despite the final $\alpha^{f}$ of the newly formed state being known. There is thus no straightforward 

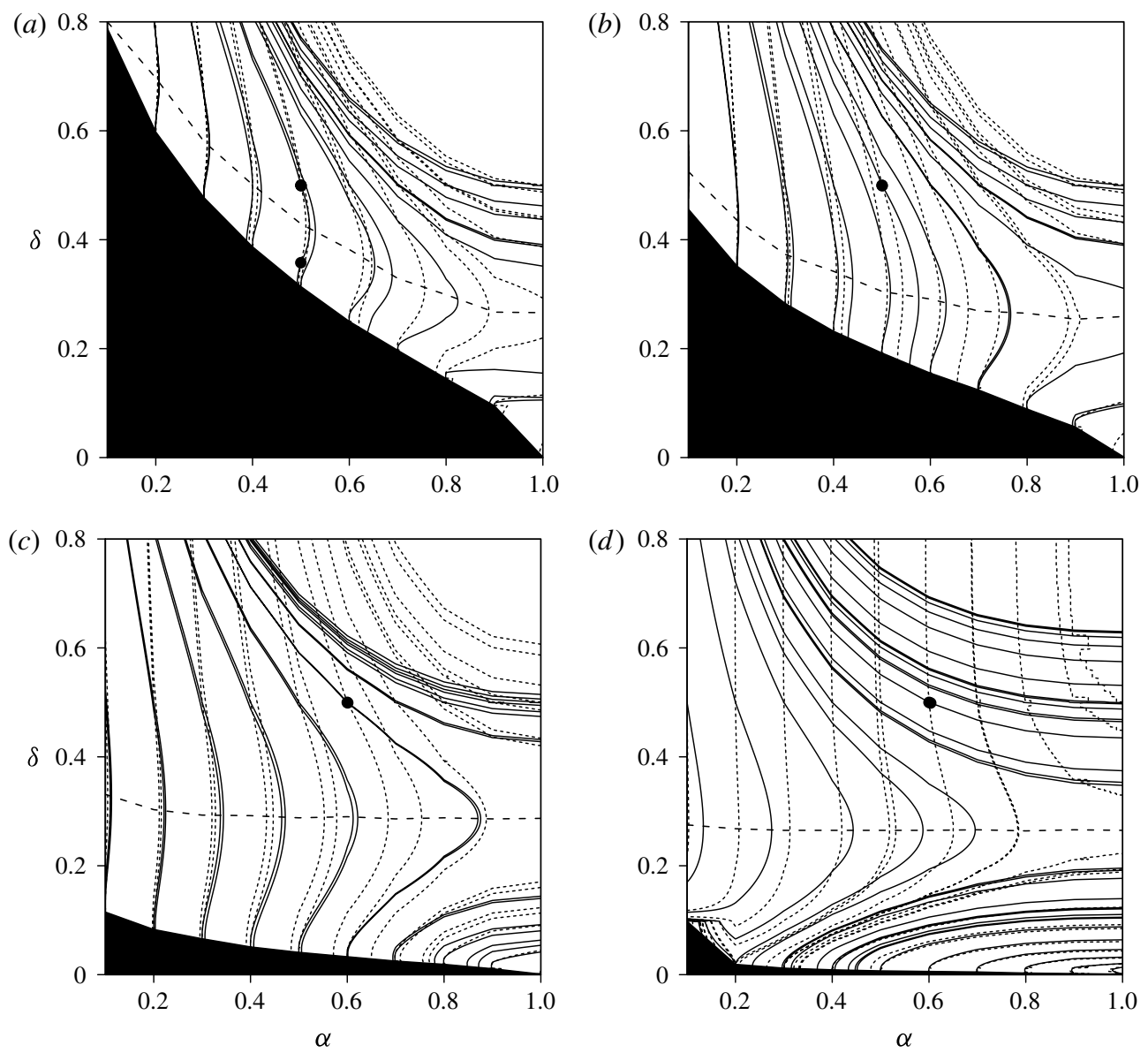

FIgURE 19. $J$ (thin line) and $E$ (dotted line) of DC states in the $\alpha-\delta$ parameter plane. We show $(a) \gamma=0.25,(b) \gamma=1,(c) \gamma=3$ and $(d) \gamma=10$. The contours extracted correspond to states with fixed $(\alpha, \delta)$, where $\alpha=0.1-1.0$ at $\Delta \alpha=0.1$ and $\delta=0.500$. States below the dashed line are unstable, and above - stable. The black dots mark the intersection points between the $J$ and $E$ contours corresponding to the same state, marking a possible complete transition from a pre-selected $\mathrm{DC}^{a}$ state. A complete transition into more than one unique $\mathrm{DC}^{b}$ state is possible when multiple black dots (i.e. multiple intersections) are present.

procedure to determine the underlying equilibrium states, even though they are likely to exist.

\section{Conclusions}

We have examined the form, properties and stability of asymmetric singlelayer quasi-geostrophic shallow-water doubly-connected rotating equilibria. In this framework, the equilibria depend on three parameters: the ratio $\gamma$ between the mean horizontal size of the vortices $L$ and the Rossby deformation length $L_{D}$, the ratio $\alpha$ between the areas of the smaller and the larger vortices, and the minimum distance $\delta$ between the two vortices. Extending the work of Polvani et al. (1989) and Dritschel (1995) we have explored the $\gamma-\alpha-\delta$ parameter space, and for each $(\gamma, \alpha)$ we have found the margin of stability $\delta=\delta_{c}$, correct to within $\Delta \delta=10^{-3}$. 

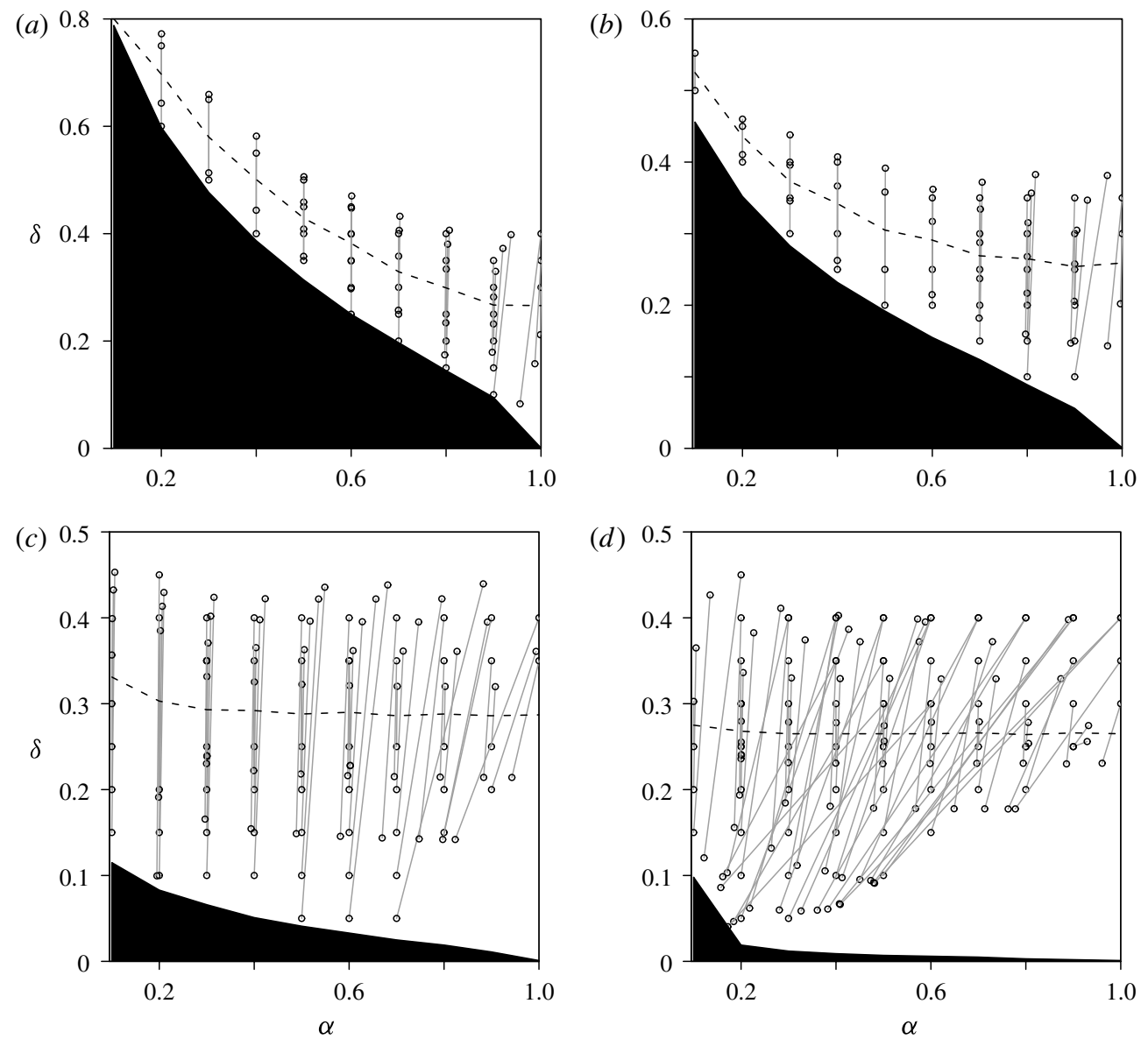

FIGURE 20. A summary of complete transitions, connected by the grey lines, possible between two DC states for $(a) \gamma=0.25$, (b) $\gamma=1$, (c) $\gamma=3$ and $(d) \gamma=10$. States below the dashed line are unstable. We only show complete transitions between unstable and stable states. Note the different scales on the $y$-axis between the figures.

By both performing a linear stability analysis and examining the nonlinear evolution of the equilibria, we have related the principal modes of linear stability to different types of evolution. We have found two principal modes of linear stability, occurring for small $\gamma$ and large $\gamma$, with a transition region between them. The small- $\gamma$ mode, absent for states with $\alpha \gtrsim 0.6$, is associated with either one, or both of the vortices getting smaller through an asymmetric instability where the smaller vortex sheds a filament from one of its tips. The large- $\gamma$ mode, present for all $\alpha$ when $\gamma \gtrsim 3$, is associated with a suppression of filamentation and with the merger of the two vortices. The transition zone between the two (also present for $\alpha \gtrsim 0.6$, but for small $\gamma \lesssim 2$ ) is characterized by a series of merging and splitting events, with the state losing area in the process, as it tries to reach a quasi-steady simply-connected state, but is unable to do so.

The effects of non-zero $\gamma$ cause the behaviour of the vortices to become $\alpha$ independent. For $\gamma \gtrsim 2$, the critical distance $\delta_{c}$ between the vortices is almost identical for all $\alpha$ (with it being slightly larger for $\alpha=0.2$ ). As $\gamma$ is decreased to the barotropic 
case $\gamma=0$, the effects of $\alpha$ become increasingly pronounced, with more asymmetric states being unstable for larger $\delta$ than symmetric ones.

Noting the fast return of the flow to a quasi-steady state after an instability has taken place, we have examined possible inviscid transitions between two doubly-connected states or, using the results of a previous study (Płotka \& Dritschel 2012), between a doubly-connected and a simply-connected state (which is described by $\gamma$ and its aspect ratio $\lambda$ ). We find that although inviscid transitions are possible, they are not the preferred path of evolution. Small- $\gamma$ states preserve neither angular impulse nor energy during unstable evolution, and a dissipative transition rather than an inviscid one is favoured in this regime. Large- $\gamma$ states, which preserve both energy and angular impulse, favour merger rather than the predicted inviscid transitions into other doubly-connected states, despite the fact that inviscid transitions between an unstable doubly-connected state and a steady simply-connected state have not been predicted to occur.

The long-time persistence of the newly formed states suggests that they oscillate around an underlying equilibrium. However, as a result of waves propagating on their boundary, it is difficult to determine these equilibria. Additionally, since we have found that there exist many robust time-dependent states especially at large $\gamma$ (which are hard to quantify), it appears that examining vortices through the concept of equilibrium is not complete. This is an important conclusion from our study, suggesting a new paradigm. Studying an initially circular vortex, for example, with a fixed area and $\gamma$, in a random (in both phase and amplitude) irrotational straining flow would provide further insight into the impact of strain on the robustness of vortices. This would also allow the examination of the stability of the large-amplitude waves travelling on the boundary of the vortex, which obey the modified $\mathrm{KdV}$ equation to leading order. These modified KdV 'breathers' have been shown to be very stable (see e.g. Alejo 2012).

To summarize, we have found that the stability of a vortex pair is not affected by the degree of its asymmetry for large vortices $\left(L \gg L_{D}\right)$, although it does affect the underlying steady state into which an unstable state transitions. We have also found that for a fixed $\alpha$ and $\delta$, smaller vortices are more likely to be unstable than larger ones, meaning that large vortices can sustain greater deformations induced by the flow of another vortex than smaller ones.

This study has focused on the quasi-geostrophic shallow-water (QGSW) 'one-anda-half-layer model' in which the lower layer is infinitely deep, i.e. $D=H_{1} / H_{2} \rightarrow 0$, where $H_{1}$ and $H_{2}$ are the depths of the upper and lower layers, respectively. However, for most oceanic and atmospheric systems the lower layer has finite depth $D=O(1)$, and so considering a two-layer system would be more realistic. Polvani et al. (1989) have argued that, in contrast to the QGSW problem, the dynamics of the two-layer system in the large- $\gamma$ limit are expected to be similar to the barotropic case, only with a rescaling of time (the 'baroclinic', vertically-varying mode becomes weak compared to the barotropic, vertically-integrated mode when $D$ is not small). Hence the large- $\gamma$ results presented here need to be interpreted with care. Polvani (1991), who examined two-layer equilibria, showed that, indeed, equilibria which do not overlap (vertically) behave similarly to the (scaled) barotropic ones, but overlapping equilibria do not. Essentially, baroclinic effects become important at short range, i.e. where vortices overlap. The vertical PV structure is also expected to play a major role; this is expected to strongly affect the relative importance of baroclinic effects, but to our knowledge has yet to be investigated in this context. Examining the two-layer system would additionally allow the study of the competition between the barotropic and 
baroclinic modes, and the energy transfer from the baroclinic to the barotropic mode in a very simple framework. This in itself is an interesting problem, especially in the oceanographic context (Ghil, Feliks \& Sushama 2002).

In addition to the two-layer problem, extending this study to the full shallow-water equations would permit one to study new effects such as spontaneous gravity-wave emission, ageostrophic effects, and the associated cyclone-anticyclone asymmetry (see e.g. Charney \& Flierl 1981; Polvani et al. 1994, and references therein). Although these equilibria are not strictly steady, theoretically-based numerical tools exist which allow the generation of equilibria which radiate such weak gravity waves that they are steady for all practical purposes (Viúdez \& Dritschel 2004).

\section{Acknowledgements}

H.P. would like to acknowledge the support of a NERC studentship. D.G.D. received support for this research from the UK Engineering and Physical Sciences Research Council (grant EP/H001794/1).

\section{Supplementary movies}

Supplementary movies are available at http://dx.doi.org/10.1017/jfm.2013.104.

\section{REFERENCES}

Alejo, M. A. 2012 Geometric breathers of the mKdV equation. Acta Appl. Maths 121, 137-155.

Carton, X. 2001 Hydrodynamical modelling of oceanic vortices. Surv. Geophys. 22, 179-263.

Cerretelli, C. \& Williamson, C. H. K. $2003 a$ The physical mechanism for vortex merging. J. Fluid Mech. 475, 41-77.

Cerretelli, C. \& Williamson, C. H. K. $2003 b$ A new family of uniform vortices related to vortex configurations before merging. J. Fluid Mech. 493, 219-229.

Charney, J. G. \& Flierl, G. R. 1981 Oceanic analogues of large-scale atmospheric motions. In Evolution of Physical Oceanography (ed. B. A. Warren \& C. Wunsch), pp. 504-548. MIT.

DEEM, G. S. \& ZABUSKY, N. J. 1978 a Stationary V-states, interactions, recurrence, and breaking. In Solitons in Action (ed. K. Longren \& A. Scott), pp. 277-293. Academic.

DeEm, G. S. \& ZABUSKy, N. J. $1978 b$ Vortex waves: stationary V-states, interactions, recurrence, and breaking. Phys. Rev. Lett. 40, 859-862.

DritsChel, D. G. 1985 The stability and energetics of corotating uniform vortices. J. Fluid Mech. 157, 95-134.

DRITSCHEL, D. G. 1986 The nonlinear evolution of rotating configurations of uniform vorticity. J. Fluid Mech. 172, 157-182.

DRITSChEL, D. G. 1988 Contour surgery: a topological reconnection scheme for extended integrations using contour dynamics. J. Comput. Phys. 77, 240-266.

DRITSCHEL, D. G. 1989 Contour dynamics and contour surgery: numerical algorithms for extended, high-resolution modelling of vortex dynamics in two-dimensional, inviscid, incompressible flows. Comput. Phys. Rep. 10, 77-146.

Dritschel, D. G. 1993 Vortex properties of two-dimensional turbulence. Phys. Fluids A 5, 984-997.

Dritschel, D. G. 1995 A general theory for two-dimensional vortex interactions. J. Fluid Mech. 293, 269-303.

Dritschel, D. G. \& Legras, B. 1991 The elliptical model of two-dimensional vortex dynamics. Part II: disturbance equations. Phys. Fluids A 3, 855-868.

Dritschel, D. G., Scott, R. K., Macaskill, C., Gottwald, G. A. \& Tran, C. V. 2008 Unifying scaling theory for vortex dynamics in two-dimenisional turbulence. Phys. Rev. Lett. 101, 094501. 
Dritschel, D. G. \& WAUgh, D. W. 1992 Quantification of the inelastic interaction of unequal vortices in two-dimensional vortex dynamics. Phys. Fluids A 4, 1737-1744.

Fontane, J. \& DRitschel, D. G. 2009 The HyperCASL algorithm: a new approach to the numerical simulation of geophysical flows. J. Comput. Phys. 228, 6411-6425.

Fornberg, B. 1977 A numerical study of 2-d turbulence. J. Comput. Phys. 25, 1-31.

Ghil, M., FeliKs, Y. \& Sushama, L. U. 2002 Baroclinic and barotropic aspects of the wind-driven ocean circulation. Physica D 167 (1-2), 1-35.

GRIFFITHS, R. W. \& Hopfinger, E. J. 1986 Experiments with baroclinic vortex pairs in a rotating fluid. J. Fluid Mech. 173, 501-518.

Griffiths, R. W. \& Hopfinger, E. J. 1987 Coalescence of geostrophic vortices. J. Fluid Mech. 178, 73-97.

Hoskins, B. J., Mcintyre, M. E. \& Robertson, R. W. 1985 On the use and significance of isentropic potential vorticity maps. Q. J. R. Meteorol Soc. 111, 877-946.

Legras, B. \& DRITsChel, D. G. 1993 Vortex stripping and the generation of high vorticity gradients in two dimensional flows. Appl. Sci. Res. 51, 445-455.

Legras, B., Dritschel, D. G. \& Caillol, P. 2001 The erosion of a distributed two-dimensional vortex in a background straining flow. J. Fluid Mech. 441, 369-398.

Luzzatto-Fegiz, P. \& Williamson, C. H. K. 2012 Determining the stability of steady two-dimensional flows through imperfect velocity-impulse diagrams. J. Fluid Mech. 706, 323-350.

Makarov, V. G. \& Kizner, Z. 2011 Stability and evolution of uniform-vorticity dipoles. J. Fluid Mech. 672, 307-325.

Makarov, V. G., Sokolovskiy, M. A. \& Kizner, Z. 2012 Doubly symmetric finite-core heton equilibria. J. Fluid Mech. 708, 397-417.

Mariotti, A., Legras, B. \& Dritschel, D. G. 1994 Vortex stripping and the erosion of coherent structures in two dimensional flows. Phys. Fluids 6, 3954-3962.

MCINTYRE, M. E. 1993 The middle atmosphere: atmospheric dynamics - some fundamentals, with observational implications. In The Use of EOS for Studies of Atmospheric Physics (ed. J. C. Gille \& G. Visconti), Proc. Intl School of Physics 'Enrico Fermi' CXV Course, pp. 313-386. North-Holland.

McWilliams, J. C. 1984 The emergence of isolated coherent vortices in turbulent flow. J. Fluid Mech. 146, 21-43.

Melander, M. V., Zabusky, N. J. \& MCWilliams, J. C. 1987 Asymmetric vortex merger in two-dimensions: which vortex is 'victorious'? Phys. Fluids 30, 2610-2612.

Meunier, P. \& Leweke, T. 2001 Three-dimensional instability during vortex merging. Phys. Fluids 13, 2747-2750.

Mitchell, T. B. \& Driscoll, C. F. 1996 Electron vortex orbits and merger. Phys. Fluids 8, $1828-1841$.

Nycander, J., Dritschel, D. G. \& Sutyrin, G. G. 1993 The dynamics of long frontal waves in the shallow water equations. Phys. Fluids A 5, 1089-1091.

Pedlosky, J. 1979 Geophysical Fluid Dynamics. Springer.

PIERREHUmbert, R. T. 1980 A family of steady, translating vortex pairs with distributed vorticity. J. Fluid Mech. 99, 129-144.

PŁotKa, H. \& DRITSChel, D. G. 2012 Quasi-geostrophic shallow-water vortex-patch equilibria and their stability. Geophys. Astrophys. Fluid 106 (6), 574-595.

Polvani, L. M. 1988 Geostrophic vortex dynamics. PhD thesis, MIT/WHOI WHOI-88-48.

Polvani, L. M. 1991 Two-layer geostrophic vortex dynamics. Part 2. Alignment and two-layer V-states. J. Fluid Mech. 225, 241-270.

Polvani, L. M. C., McWilliams, J., Spall, M. A. \& Ford, R. 1994 The coherent structures of shallow-water turbulence: deformation-radius effects, cyclone/anticyclone asymmetry and gravity-wave generation. Chaos 4 (2), 177-186.

Polvani, L. M., Zabusky, N. J. \& Flierl, G. R. 1989 Two-layer geostrophic vortex dynamics. Part 1. Upper-layer V-states and merger. J. Fluid Mech. 205, 215-242.

Saffman, P. G. 1992 Vortex Dynamics. Cambridge University Press. 
Saffman, P. G. \& Szeto, R. 1980 Equilibrium shapes of a pair of equal uniform vortices. Phys. Fluids 23, 2339-2342.

Santangelo, R., Benzi, R. \& Legras, B. 1989 The generation of vortices in high-resolution, two-dimensional decaying turbulence and the influence of initial conditions on the breaking of self-similarity. Phys. Fluids A 1, 1027-1034.

Scott, R. K. \& Polvani, L. M. 2008 Equatorial superrotation in shallow atmospheres. Geophys. Res. Lett. 35, L24202.

Trieling, R. R., Fuentes, O. U. \& VAn Heijst, G. J. 2005 Interaction of two unequal corotating vortices. Phys. Fluids 17, 087103.

VIÚDEZ, Á. \& DRITSChEL, D. G. 2004 Dynamic potential vorticity initialization and the diagnosis of mesoscale motion. J. Phys. Oceanogr. 34, 2761-2773.

Waugh, D. W. 1992 The efficiency of symmetric vortex merger. Phys. Fluids A 4, 1745-1758.

Waugh, D. W. \& Dritschel, D. G. 1991 The stability of filamentary vorticity in two-dimensional geophysical vortex-dynamics models. J. Fluid Mech. 231, 575-598.

Wu, H. M., Overman, E. A. II \& Zabusky, N. J. 1985 Steady-state solutions of the Euler equations in two dimensions: rotating and translating V-states with limiting cases. I. Numerical algorithms and results. J. Comput. Phys. 53, 42-71.

YASUDA, I. 1995 Geostrophic vortex merger and streamer development in the ocean with special reference to the merger of Kuroshio warm core rings. J. Phys. Oceanogr. 25, 979-996.

YASUDA, I. \& FlieRL, G. R. 1995 Two-dimensional asymmetric vortex merger: contour dynamics experiment. J. Phys. Oceanogr. 51, 145-170.

Zabusky, N. J., Hughes, M. H. \& Roberts, K. V. 1979 Contour dynamics for the Euler equations in two dimensions. J. Comput. Phys. 30, 96-106. 